ANNUITY VALUATION WITH DEPENDENT MORTALITY

\author{
by \\ Edward W. Frees, University of Wisconsin - Madison \\ Jacques Carriere, University of Manitoba and \\ Emiliano Valdez, University of Wisconsin - Madison \\ May, 1995
}

Keywords and Phrases: Survival Analysis, Bivariate Distributions, Copulas, Annuity Pricing, Adverse Selection.
Acknowledgements: The research of Jacques Carriere and Emiliano Valdez was supported by the Society of Actuaries. The research of Edward Frees was partially supported by a professorship funded by the Time Insurance Company and the Social Security Administration, Grant No. 10-P-98353-5-01.

Please address correspondeace to:

Edward W. Frees

School of Business

975 University Avenue

Madison, Wisconsin 53706

jfrees@bus.wisc.edu 


\title{
Annuity Valuation with Dependent Mortality
}

\begin{abstract}
Annuities are contractual guarantees, issued by insurance companies, pension plans, and government retirement systems, that offer promises to provide periodic income over the lifetime of individuals. It is well-known how to use univariate models of survivorship for valuing annuities. However, standard industry practice assumes independence of lives when valuing annuities where the promise is based on more than one life.

This paper investigates the use of models of dependent mortality for determining annuity values. We discuss a broad class of parametric models using a bivariate survivorship function called a copula. Using data from a large insurance company to illustrate our methods, we calculate maximum likelihood estimates to calibrate the model.

The estimation results show strong positive dependence between joint lives. This statistically significant result translates into real economic significance. That is, there is an approximate five percent reduction in annuity values when dependent mortality models are used compared to the standard models that assume independence. We show that the results are robust in terms of the choice of parametric family of distribution functions.
\end{abstract}




\section{Annuity Valuation with Dependent Mortality}

\section{Introduction}

Financial service organizations offer contractual promises to provide periodic level incomes over the lifetime of individuals. These contracts, called annuities, typically provide a level monthly amount payable until the death of a named individual, called an annuitant. Annuity obligations are offered by insurance companies, pension and other employee benefit funds, and state and federal retirement systems. To illustrate the importance of these obligations, U.S. insurance companies alone made $\$ 40.3$ billions in annuity payments in 1993 (1994 Life Insurance Fact Book).

An important variation of the standard life annuity is the joint and last-survivor annuity. Under this contract, periodic level payments are made until the last of a group of individuals dies. To illustrate, a prime example of a group is a married couple, where the last-survivor annuity pays as long as either spouse survives. Many variations are offered in the marketplace, including a joint and $50 \%$ annuity that pays a level amount while both annuitants survive with a fifty percent reduction of that amount upon the death of one annuitant.

Valuation of life annuities depends upon (i) the time value of money and (ii) probability of survivorship of the annuitants. The time value of money is important because annuity payments are made in the future with respect to the valuation of the annuity obligation. However, conditional on survivorship of the annuitants, these payments can be valued using standard theory from financial economics such as the term structure of interest rates. We do not pursue this aspect of annuity valuation here. Instead, we apply the traditional approach of assuming a constant discount rate.

In this article, we focus on estimating the probability of joint survivorship of two annuitants. Estimation of survival probabilities for more than two annuitants can be done by direct extensions of the methods of this paper. We focus on two annuitants because the number and amounts of annuity contracts issued with more than two annuitants is small compared to the case of two annuitants.

Traditionally, estimation of joint survival probabilities of a pair has been done by assuming independence of lives. With this assumption, the probability of joint survival is the product of the probability of survival of each life. This assumption reduces the joint estimation to a single life estimation problem. Estimation of the probability of survivorship of a single annuitant is a well developed area (see, for example, Elandt-Johnson and Johnson, 1980, or Cox and Oakes, 1990).

However, several empirical studies of joint lives in non-commercial contexts have established that 
survival of pairs are not independent events. To illustrate, Hougaard, Harvald and Holm (1992) analyzed the joint survival of Danish twins born between 1881 and 1930. In epidemiological studies of diseases, analysis of twins data is useful because of the ability to control for heredity factors within each pair of twins. Another type of empirical study involves measuring the impact on mortality induced by the mortality of one's spouse. Parkes et al (1969) and Ward (1976) provide early examples of this impact, often called the "broken heart" syndrome. A more recent study, with references to many other works, is by Jagger and Sutton (1991).

There are several ways to model the impact of survivorship of one life upon another. For example, as in Jagger and Sutton (1991), the question of increased mortality after an event such as the death of a spouse is well-suited to a survival model called proportional hazards that allows for timevarying explanatory variables. This type of model was also used by Hougaard et al (1992) to assess the generations effect of studying twins over a long period of time.

Classical models of dependent lives are called "common shock" models, see, for example, Marshall and Olkin (1967). These models assume that the dependence of lives arises from an exogenous event that is common to each life. For example, in lifetime analysis this shock may be an accident or the onslaught of a contagious disease. Although there are many other types of dependencies in human lifetimes that are not captured by shock models, their particularly simple form turns out to be convenient for annuity valuation purposes. These models are further discussed in Section 6 below.

Other parametric bivariate survival models include the "frailty" models described by Oakes (1989), the mixture models of Marshall and Olkin (1988) and the "copula" models, as described in Genest and McKay (1986). Nonparametric estimation of bivariate survival estimation has been summarized by Pruitt (1993). Because of the several complications that appear in annuity data, we focus on the copula models. These models provide tractable parametric models of the bivariate distribution and are described in more detail in Section 3.

To calibrate our models, we consider data from a large Canadian insurance company. We study the mortality experience from observing approximately fifteen thousand policies over a five year period including 1989 through 1993. Further description of the sources and characteristics of the data is in Section 2 .

Because of the nature of our data and our interest in annuity valuation, this paper differs from other empirical studies of bivariate distributions in several aspects. First, our data sampling period, five years, is much shorter than other lifetime studies (for example, the Danish twins data were observed over a one hundred ten year period from 1881 to 1990 ). Thus, we need not discuss cohort effects of morality 
as in that study. However, because of the short time frame of our observation period, our data are (right) censored in that most policyholders survived through the end of the observation period. Further, our data are (left) truncated in that policyholders who had died prior to the beginning of the study were not available for analysis. This complication is called "left-truncation with right-censoring" in survival analysis (see, for example, Cox and Oakes, 1990, p 177).

Second, in this paper, the scientific interest is different. Works that study the "broken heart" syndrome often wish to establish predictive models, that is, identifying an event such as the death of a spouse to improve the predictions of the probability of death. Epidemiological studies often wish to isolate explanatory variables that induce the onslaught of a certain disease or infection. Our interest lies in the valuation of annuity contracts. As such, we are interested in assessing the strength of dependence and the effects of the dependence on contract values. In particular, the choice of the model of bivariate dependence is influenced by the desire for simplicity in our annuity valuation procedures.

Third, reporting mechanisms for industry data tend to be different when compared to population data that might be gathered by the U.S. Census Bureau or data from a carefully designed clinical trial. For industry, two important issues are the mortality patterns within a contractual guarantee period and the reporting of the first death for some contracts. Some joint-life contracts offer a guarantee of annuity payments, typically over a period of five or ten years from contract initiation. Thus, there is no economic incentive for reporting a death within the guarantee period. Alternatively, those that elect the guarantee option may exhibit higher mortality than those who do not. Further, for joint and last-survivor policies, payments are made until the second, or last, of the pair dies. Thus, although policyholders should notify the insurance company of a change in the mortality status of annuitants, there is no economic purpose for reporting the death of the first annuitant. These two issues are addressed in greater detail in Section 5.

Here is a summary for the rest of the paper. In Section 2 we introduce the data and in Section 3 we discuss models of dependence. Section 4 summarizes the effects of dependence on annuity values and Section 5 addresses the problem of underreporting. Section 6 examines the robustness of choice of parametric families. This is done by considering alternative marginal distribution and an alternative bivariate distribution function, the common shock model. We close in Section 7 with some concluding remarks. 


\section{Data Characteristics}

In this paper, we analyze mortality patterns based on information from 14,947 contracts in force with a large Canadian insurer over the period December 29, 1988 through December 31, 1993. These contracts are joint and last-survivor annuities that were in the payout status over the observation period.

For each contract, we have the date of birth, date of death (if applicable), date of contract initiation and sex of each annuitant. Table 1 presents the frequency distribution of annuitants by sex, entry age and mortality status group. Entry age is defined to be the age at which the annuitant entered the study and was computed from the date of birth and contract initiation date. For mortality status, we classified annuitants according to whether or not they survived until the end of the observation period. In addition to the dates discussed above, we also have the date that the annuity guarantee expired (if applicable). This will be discussed further in Section 5 .

Table 1 shows that there were roughly an equal number of males and females in our study, 14,933 males and 14,961 females. We also see that there is roughly three times as many male deaths as female deaths. This is in part due to the higher average entry age for males than for females, which turns out to be approximately 68 for males and 65 for females. It also suggests higher mortality rates for males than for females.

\begin{tabular}{|c|c|c|c|}
\hline \multirow[t]{2}{*}{ Entry Age } & \multicolumn{2}{|c|}{ Mortality Status } & \multirow[b]{2}{*}{ Total } \\
\hline & Survive & Death & \\
\hline \multicolumn{4}{|l|}{ Males } \\
\hline Less than 60 & 1,170 & 42 & 1,212 \\
\hline $60-70$ & 7,620 & 534 & 8,154 \\
\hline $70-80$ & 4,355 & 806 & 5,161 \\
\hline Greater than 80 & 229 & 177 & 406 \\
\hline Total & 13,374 & 1,559 & 14,933 \\
\hline \multicolumn{4}{|l|}{ Females } \\
\hline Less than $60^{\circ}$ & 2,962 & 30 & 2,992 \\
\hline $60-70$ & 8,222 & 239 & 8,461 \\
\hline $70-80$ & 3,014 & 245 & 3,259 \\
\hline Greater than 80 & 186 & 63 & 249 \\
\hline Total & 14,384 & 577 & 14,961 \\
\hline
\end{tabular}


Figure 1 displays a graphical summary of the distribution of lifetimes for our 14,933 male annuitants. Because our data primarily concerns policyholders who are at least middle-aged, we present distribution functions that are conditional on survival to age forty. The jagged function in Figure 1 is the Kaplan-Meier product limit estimator of the distribution function. We use this as our baseline estimator of the distribution function because it is the nonparametric maximum likelihood estimator. See, for example, Elandt-Johnson and Johnson (1980) or Cox and Oakes (1990) for an introduction and further discussion of the properties of this estimator. We see from the Kaplan-Meier estimator in Figure 1, for example, that the median age at death is approximately 82 years. The $25^{\text {th }}$ and $75^{\text {th }}$ percentiles are approximately 68 and 90 years, respectively.

Superimposed in Figure 1 is a smooth curve that was fit using the Gompertz distribution. The Gompertz distribution function can be expressed as:

$$
F(x)=1-\exp \left(\mathrm{e}^{-m / \sigma}\left(1-\mathrm{e}^{x / \sigma}\right)\right)
$$

where the mode, $m$, and the scale measure, $\sigma$, are parameters of the distribution. To actuaries, the familiar Gompertz force of mortality, or hazard rate, is $\mu_{x}=\mathrm{F}^{\prime}(x) /(1-\mathrm{F}(x))=\mathrm{B} c^{x}$, that yields

$$
\mathrm{F}(x)=1-\exp \left((\mathrm{B} / \ln c)\left(1-c^{x}\right)\right)
$$

However, with the transformations $\mathrm{B} / \mathrm{ln} c=\mathrm{e}^{-m / \sigma}$ and $c=\mathrm{e}^{1 / \sigma}$, we see that equation (2.1) is simply a reparameterized version of the usual expression for the Gompertz distribution. As pointed out by Carriere (1994), equation (2.1) is convenient for estimation purposes.

Figure 1 shows that the Gompertz fit closely replicates the nonparametric Kaplan-Meier fit. The main advantage of the Gompertz fit is that only two parameter estimates are necessary to reproduce this curve. For male annuitants, the estimates turn out to be $\hat{h} \approx 86.4$ and $\hat{\sigma} \approx 9.8$ years. However, to reproduce the Kaplan-Meier estimate, all 14,933 male lifetimes would be needed because of the continuous nature of our data. The parsimonious representation provided by the Gompertz curve is particularly important for the annuity calculations in Section 4.

Of course, to achieve a parsimonious representation of a lifetime distribution, there are many other families of distribution functions that could be used. To illustrate, Figure 2 shows a fitted Weibull distribution function for the male annuitants with the Kaplan-Meier curve superimposed for reference. 
Distribution

Function

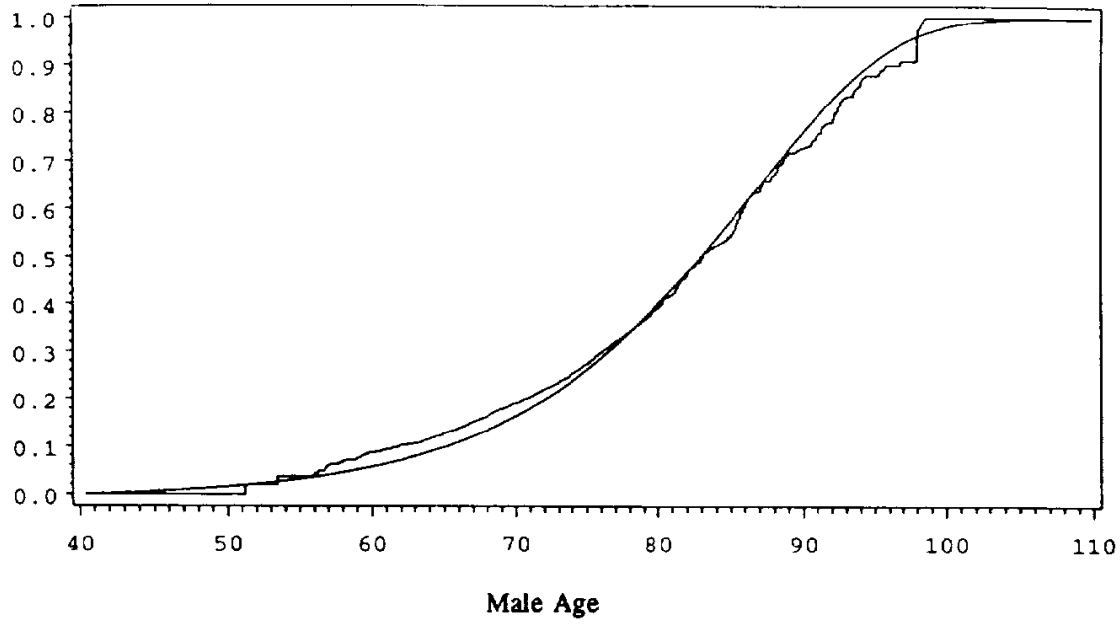

Figure 1. Gompertz and Kaplan-Meier Fitted Male Distribution Functions. The Gompertz curre is smooth, the Kaplan-Meier is jagged. The distribution is conditional on survival to age forty.

Distribution

Function

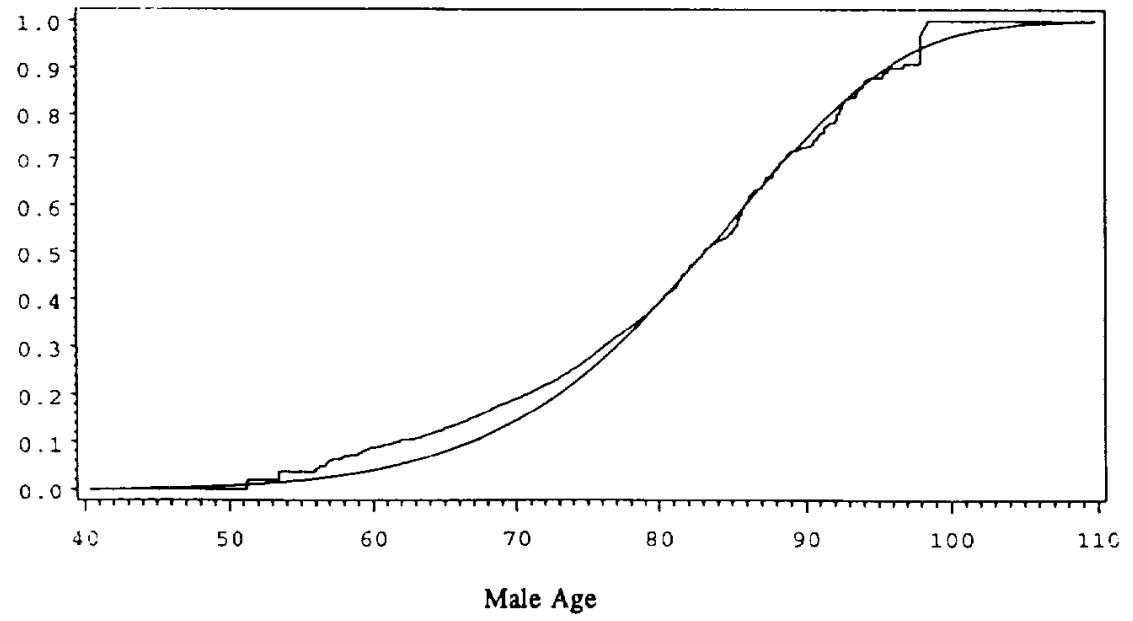

Figure 2. Weibull and Kaplan-Meier Fitted Male Distribution Functions. The Weibull curve is smooth, the Kaplan-Meier is jagged. The distribution is conditional on survival to age forty. 
The fit is close, although the Gompertz may be a better approximation of the Kaplan-Meier. For purposes of annuity valuation, it turns out that any parametric representation of the lifetime distribution suffices. For our data set of older policyholders, the Gompertz distribution seems to provide an adequate fit. There is certainly a long history of fitting Gompertz distributions to the mortality of human populations, as described by Carriere (1994) and Pollard and Volkovics (1993).

To understand some aspects of the relationship between male and female mortality, Figure 3 provides a quantile-quantile $(q-q)$ plot that compares the male and female lifetime distributions. The $q-q$ plot is a scatter plot of selected quantiles, or percentiles, from the male distribution versus the corresponding quantiles from the female distribution. Here, we used quantiles calculated from the KaplanMeier fitted distributions. The $q-q$ plot allows us to compare medians, and much more. For example, Figure 3 shows that the median male age of 82 corresponds to a median female age of 88 . Similarly, the $25^{\text {th }}$ and $75^{\text {th }}$ percentiles for males, 68 and 90 , correspond to female ages 80 and 98 , respectively. Figure 3 shows that the entire distribution of female ages at death is higher than the male distribution.

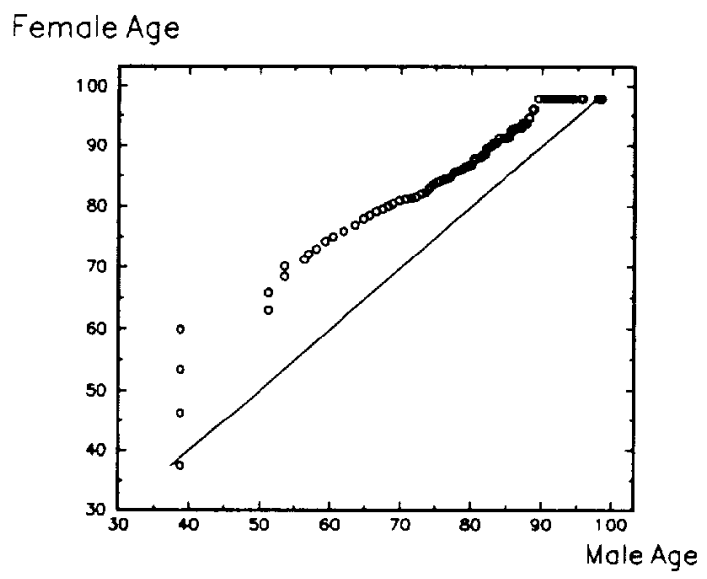

Figure 3. Quantile-Quantile Plot of Female versus Male Ages. There are one hundred percentiles plotted, where each percentile comes from the univariate Kaplan-Meier estimate of the distribution function. The solid line corresponds to male equal to female age. Because virtually all points are above the line, each female quantile exceeds the corresponding male quantile. 
To provide background on the estimation procedures that Figures 1 through 3 are based on, we now give details on the limitations of our data, including truncation and censoring. Consider the bivariate ages-at-death random vector $(X, Y)$ where $X$ and $Y$ represent the ages at death of the primary and secondary annuitant, respectively. In joint annuity contracts, one annuitant is usually designated as "primary" and the other "secondary" because some contracts provide for a reduced payout upon the death of the primary annuitant. An example of this is an annuity provided by a firm to an employee. This distinction turns out to be unimportant in our analysis of the data.

Industry data are truncated in the sense that data are only observed after a contract has been entered into by policyholders. Thus, we use standard notation and let $x$ and $y$ be the contract initiation ages of the primary and secondary annuitants, respectively. Further, for our data set, we observed the joint annuity contract if both annuitants were alive at the beginning of the observation period or if the annuitants entered the study during the observation period. With $t_{0}$ as the time of contract initiation, we define

$$
a=\max \left(12 / 29 / 88-t_{0}, 0\right)
$$

to be the time from contraction initiation to the beginning of the observation period. Thus, $x+a$ and $y+a$ are the entry ages of the primary and secondary annuitants, respectively. Under our left-truncation, we observe the contract only if $X>x+a$ and $Y>y+a$.

Our data was also censored from the right. Let $b=1 / 1 / 94-\max (12 / 29 / 88, b)$ denote the length of time that the policy was under observation. Denote $T_{1}=X-x-a$ and $T_{2}=Y-y-a$ to be the future annuitant lifetimes. Then, for $j=1,2$, we observed $T_{j}^{*}=\min \left(T_{j}, b\right)$, the censored future lifetime, and $\delta_{j}$, a variable to indicate whether censoring has occurred. That is, $\delta_{j}$ is defined to be one if $T_{j}>b$ and zero otherwise.

Using this notation, our full data set consists of $\left\{T_{i j}^{*}, \delta_{i j}\right\}, j=1,2$, and $i=1, \ldots, 14947$. That is, there are a total of 29,894 ( $=2 \times 14947)$ univariate observations. Univariate distributions were fit for each sex using maximum likelihood techniques to produce the fitted parametric curves. We do not present the details here because the more complex bivariate situation is discussed in the next section. As mentioned above, the nonparametric curves were fit using the standard Kaplan-Meier product limit estimator.

Beginning in the next section, we focus our estimation procedures using bivariate observations, that is, observations of the joint mortality of both annuitants. For our data, we have 22 contracts where both annuitants are male, 36 contracts where both annuitants are female, and 14,889 contracts where one annuitant is male and the other female. Because of the preponderance of data in the third category, we focus our attention on male-female joint annuity mortality. Henceforth, we refer to $x$ as the male life and 
$y$ as the female life. Of course, the estimation techniques that we introduce could also be applied to the other two categories.

A classical nonparametric measure of dependence is Spearman's rank correlation. Appendix E shows how we can use this measure when the data are left-truncated, right-censored and not identically distributed. The test of independence that we present assumes that the law of mortality is known for individual lives. Obviously, our knowledge of this law for annuity products is substantial but not perfect and so caution must be used when using this method. Using this technique we found that the correlation was 0.41 and that a $95 \%$ confidence interval is $(0.28,0.55)$. If the lives were independent, then the correlation is zero. Therefore, this crude preliminary analysis suggests that the lifetimes are dependent. In subsequent sections, we will corroborate this analysis using classical maximum likelihood techniques.

\section{Models of Dependence}

\subsection{Bivariate Distributions}

In this paper, we express our bivariate distributions using a function called a copula. Consider a bivariate age-at-death random vector $(X, Y)$ with distribution function $H$, that is, $H(x, y)=\operatorname{Prob}(X \leq x$, $Y \leq y)$. Let $F_{1}$ and $F_{2}$ denote the respective marginal distribution functions so that $F_{1}(x)=H(x, \infty)$ and $F_{2}(y)=H(\infty, y)$. We consider bivariate distribution functions of the form

$$
H(x, y)=C\left(F_{1}(x), F_{2}(y)\right)
$$

Here, $C$ is a real-valued function called a copula. Copulas are bivariate distributions with uniform marginal distributions.

Copulas are useful because they provide a link between the marginal distributions and the bivariate distribution. From equation (3.1), it is clear that if $F_{1}, F_{2}$ and $C$ are known, then $H$ can be determined. Sklar (1959) proved a converse: if $H$ is known and if $F_{1}$ and $F_{2}$ are known and continuous, then $C$ is uniquely determined. In this sense, $C$ "couples" the marginal distributions to the bivariate distribution.

There are many possible choices of the copula function. In this paper, we focus on a one parameter family due to Frank (1979) that can be expressed as

$$
C(u, v)=\ln \left(1+\frac{\left(e^{\alpha u}-1\right)\left(e^{\alpha v}-1\right)}{e^{\alpha}-1}\right) / \alpha
$$


Advantages of this family have been presented by Nelsen (1986) and Genest (1987). The Frank, Genest and Nelsen papers present the copula in terms of the parameter $\gamma=e^{\alpha}$. Similarly to the case of the univariate Gompertz distribution, we work with the reparameterized version in equation (3.2). This transformation turns out to be more convenient for estimation purposes.

The parameter $\alpha$ captures the dependence between $X$ and $Y$. The case of independence corresponds to $\alpha=0$. This is because it can easily be shown, from equation (3.2), that $\lim _{\alpha \rightarrow 0} \mathrm{C}(u, v)$ $=u v$. Thus, the bivariate distribution function is the product of marginal uniform distributions. In addition to the dependence parameter $\alpha$, we also present Spearman's correlation coefficient $\rho(\alpha)$. Spearman's correlation coefficient is a nonparametric measure, defined to be the ordinary Pearson correlation coefficient after taking a (marginal) uniform transformation of each random variable. Here, $\rho(\alpha)$ is a straightforward function of $\alpha$ that Nelsen (1986) showed to be

$$
\rho(\alpha)=1-12\left(D_{2}(-\alpha)-D_{1}(-\alpha)\right) / \alpha
$$

where $\mathrm{D}_{k}(x)=k x^{-k} \int_{0}^{x} t^{k}\left(e^{t}-1\right)^{-1} d t, k=1,2$, is called the Debye function.

To complete our specification of the bivariate distribution, we assume that each marginal distribution is Gompertz. Thus, using equation (2.1), we assume

$$
F_{j}(x)=1-\exp \left(\exp \left(-m_{j} / \sigma_{j}\right)\left(1-\exp \left(x / \sigma_{j}\right)\right)\right), j=1,2
$$

Our model is then specified by equations (3.1), (3.2) and (3.4). This model has five parameters which can be represented by the vector

$$
\theta=\left(m_{1}, \sigma_{1}, m_{2}, \sigma_{2}, \alpha\right)^{\prime}
$$

\subsection{Estimation Results}

Using the left-truncated, right-censored data described in Section 2, we estimated the model described in Subsection 3.1. The method of estimation is maximum likelihood; the details are presented in Subsection 3.3 below. The results of the estimation are summarized in Table 2. 


\begin{tabular}{|c|c|c|c|c|}
\hline \multirow[b]{2}{*}{ Parameter } & \multicolumn{2}{|c|}{ Bivariate Distribution } & \multicolumn{2}{|c|}{ Univariate Distributions } \\
\hline & Estimate & Standard Error & Estimate & Standard Error \\
\hline$m_{1}$ & 85.82 & 0.26 & 86.38 & 0.26 \\
\hline$\sigma_{1}$ & 9.98 & 0.40 & 9.83 & 0.37 \\
\hline$m_{2}$ & 89.40 & 0.48 & 92.17 & 0.59 \\
\hline$\sigma_{2}$ & 8.12 & 0.34 & 8.11 & 0.38 \\
\hline$\alpha$ & -3.367 & 0.346 & Nor Applicable & No Applicable \\
\hline
\end{tabular}

Table 2 shows that the "average," or modal, age at death was approximately fours years later for females than males. The estimates of variability were roughly the same. Using equation (3.3), the estimate of the dependence parameter can be converted to a correlation estimate. This turns out to be $\rho(\hat{\alpha})$ $=\rho(-3.367)=0.49$. Recall that Spearman's correlation, like Pearson's correlation, is bounded by -1 and 1 with a correlation of zero implying no relationship. A value of $\rho(\hat{\alpha})=0.49$ indicates a strong statistical dependence. This is because a rough 95\% confidence interval for $\alpha$ is $\hat{\alpha} \pm 1.96 \operatorname{se}(\hat{\alpha})=-3.367 \pm 1.96$ $(0.346)=(-4.045,-2.689)$. Translated into the correlation scale, a $95 \%$ confidence interval of Spearman's correlation is $(0.41,0.56)$.

The Table 2 parameter estimates can be directly used to value annuities. Some readers may wish to skip directly to Section $\mathbf{4}$ where we analyze the effects of mortality dependence on annuity valuation. Others may be interested in estimating the parameters for their own block of business and hence, in the estimation details which we now present.

\subsection{Maximum Likelihood Estimation}

We now develop the likelihood function to be maximized. Having developed the likelihood function, standard function maximization routines will yield the maximum likelihood estimates. Because our sampling satisfies standard regularity conditions (see, for example, Serfling, 1980), we can easily get asymptotic normality and subsequent standard errors for the estimates.

In our development, we will need the following partial derivatives: (i) $\mathrm{H}_{1}(x, y)=\partial \mathrm{H}(x, y) / \partial x$, (ii) $\mathrm{H}_{2}(x, y)=\partial \mathrm{H}(x, y) / \partial y$, and (iii) $\mathrm{h}(x, y)=\partial^{2} \mathrm{H}(x, y) / \partial x \partial y$. Our assumption of Gompertz marginals and Frank's copula assures that these derivatives exist. Their explicit representation in terms of the vector of parameters is given in Appendix A.

To develop the likelihood function, we first consider truncated observations. Recall that the future 
lifetime random variables $T_{1}=X-x-a$ and $T_{2}=Y-y-a$ are observed only if $T_{1}>0$ and $T_{2}>0$. Therefore, define the conditional distribution function of $T_{1}$ and $T_{2}$ as

$$
\begin{gathered}
H_{\mathrm{T}}\left(t_{1}, t_{2}\right)=\operatorname{Prob}\left(T_{1} \leq t_{1}, T_{2} \leq t_{2} \mid T_{1}, T_{2} \text { are observed }\right)=\frac{\operatorname{Prob}\left(0<T_{1} \leq t_{1}, 0<T_{2} \leq t_{2}\right)}{\operatorname{Prob}\left(T_{1}>0, T_{2}>0\right)} \\
=\frac{\mathrm{H}\left(x+a+t_{1}, y+a+t_{2}\right)-\mathrm{H}\left(x+a, y+a+t_{2}\right)-\mathrm{H}\left(x+a+t_{1}, y+a\right)+\mathrm{H}(x+a, y+a)}{1-\mathrm{H}(x+a, \infty)-\mathrm{H}\left(\infty_{2} y+a\right)+\mathrm{H}(x+a, y+a)} .
\end{gathered}
$$

Turning now to the case of right-censoring, recall that $T_{j}^{*}=\min \left(T_{j}, b\right)$. There are four types of censoring that may occur. The lifetimes may be: (i) both uncensored, (ii) the first uncensored and the second censored, (iii) the first censored and the second uncensored and (iv) both censored. We handle each type in turn.

1. If both lifetimes are uncensored, then we may assume $t_{1}<b$ and $t_{2}<b$. In this case, we have $\delta_{1}=0$ and $\delta_{2}=0$ and

$$
\begin{gathered}
\operatorname{Prob}\left(T_{1}^{*}<t_{1}, T_{2}^{*}<t_{2} \mid T_{1}^{*}, T_{2}^{*}\right. \text { are observed) } \\
=\operatorname{Prob}\left(\min \left(T_{1}, b\right)<t_{1}, \min \left(T_{2}, b\right)<t_{2} \mid T_{1}>0, T_{2}>0\right)=\mathrm{H}_{T}\left(t_{1}, t_{2}\right) .
\end{gathered}
$$

Thus, using equation (3.6), the contribution to the likelihood function is:

$$
\frac{\partial^{2} H_{1}\left(t_{1}, t_{2}\right)}{\partial x_{1} \partial t_{2}}=\frac{h\left(x+a+t_{1}, y+a+t_{2}\right)}{1-H(x+a, \infty)-H(\infty, y+a)+H(x+a, y+a)} .
$$

2. If the first lifetime is uncensored and the second is censored, then we may assume $t_{1}<b$ and $t_{2} \geq b$. In this case, we have $\delta_{1}=0$ and $\delta_{2}=1$ and

$$
\begin{gathered}
\operatorname{Prob}\left(T_{1}^{*}<t_{1}, T_{2}^{*}=b \mid T_{1}^{*}, T_{2}^{*} \text { are observed }\right) \\
=\operatorname{Prob}\left(T_{1}<t_{1}, T_{2} \geq b \mid T_{1}>0, T_{2}>0\right)=\mathrm{H}_{T}\left(t_{1}, \infty\right)-\mathrm{H}_{T}\left(t_{1}, b\right) .
\end{gathered}
$$

Thus, the contribution to the likelihood function is:

$$
\frac{\partial\left(\mathrm{H}_{\mathrm{T}}\left(t_{1}, \infty\right)-\mathrm{H}_{\mathrm{T}}\left(t_{1}, b\right)\right)}{\partial t_{1}}=\frac{\mathrm{H}_{1}\left(x+a+t_{1}, \infty\right)-\mathrm{H}_{1}\left(x+a+t_{1}, y+a+b\right)}{1-\mathrm{H}(x+a, \infty)-\mathrm{H}(\infty, y+a)+\mathrm{H}(x+a, y+a)} .
$$


3. If the first lifetime is censored and the second is uncensored, then we may assume $t_{1} \geq b$ and $t_{2}<b$. This case is similar to case (2), thus $\delta_{1}=1$ and $\delta_{2}=0$ and the contribution to the likelihood function is:

$$
\frac{H_{2}\left(\infty, y+a+t_{2}\right)-H_{2}\left(x+a+b, y+a+t_{2}\right)}{1-H(x+a, \infty)-H(\infty, y+a)+H(x+a, y+a)} .
$$

4. If both lifetimes are censored, then we may assume $t_{1} \geq b$ and $t_{2} \geq b$. In this case, we have $\delta_{1}=1, \delta_{2}=1$ and contribution to the likelihood function is:

$$
\operatorname{Prob}\left(T_{1}^{*}=b, T_{2}^{*}=b \mid T_{1}^{*}, T_{2}^{*} \text { are observed }\right)=\frac{1-H(x+a+b, \infty)-H(\infty, y+a+b)+H(x+a+b, y+a+b)}{1-H(x+a, \infty)-H(\infty, y+a)+H(x+a, y+a)} .
$$

Combining equations (3.7) through (3.10), we can express the logarithm of the likelihood function for a single observation as

$$
\begin{gathered}
\ln L\left(x, y, t_{1}, t_{2}, \delta_{1}, \delta_{2} a, b\right)=\left(1-\delta_{1}\right)\left(1-\delta_{2}\right) \ln \mathrm{h}\left(x+a+t_{1}, y+a+t_{2}\right) \\
+\left(1-\delta_{1}\right) \delta_{2} \ln \left(\mathrm{H}_{1}\left(x+a+t_{1}, \infty\right)-\mathrm{H}_{1}\left(x+a+t_{1}, y+a+b\right)\right)+\delta_{1}\left(1-\delta_{2}\right) \ln \left(\mathrm{H}_{2}\left(\infty, y+a+t_{2}\right)-\mathrm{H}_{2}\left(x+a+b, y+a+t_{2}\right)\right) \\
+\delta_{1} \delta_{2} \ln (1-\mathrm{H}(x+a+b, \infty)-\mathrm{H}(\infty, y+a+b)+\mathrm{H}(x+a+b, y+a+b)) \\
-\ln (1-\mathrm{H}(x+a, \infty)-\mathrm{H}(\infty, y+a)+\mathrm{H}(x+a, y+a)) .
\end{gathered}
$$

Using equation (3.11), the log-likelihood for the data set can be calculated as

$$
\ln \varphi=\sum_{i=1}^{n} \ln L\left(x_{p}, y_{i}, t_{1 p}, t_{2 p} \delta_{1 p} \delta_{2 p} a_{p}, b_{j}\right) \text {. }
$$

The maximum likelihood estimator of $\theta$ is the value that maximizes $\ln \mathscr{\Psi}$. Standard maximum likelihood estimation theory provides that $n^{1 / 2}(\theta-\theta)$ is asymptotically normally distributed with mean zero and variance-covariance $V_{n}(\theta)=-\left(n^{-1} \partial^{2}(\ln \mathscr{L}) /\left(\partial \theta \partial \theta^{\prime}\right)\right)^{-1}$. The variance-covariance matrix $V_{n}(\theta)$ can be consistently estimated using $V_{n}(\theta)$, which is an output from standard function maximization routines. The standard error of each parameter estimate in may be determined by the square root of the corresponding diagonal element of $V_{n}(\theta)$ divided by $n$. 


\section{Effects of Dependence on Annuity Values}

As described in Section 1, the main purpose of this paper is to assess the effects of our models of dependent mortality on annuity values. We use the basic model of annuity valuation described in Chapter 8 of Bowers et al (1986). To this end, in our illustrations below we assume a constant effective interest rate $i$ with associated discount rate $v=1 /(1+i)$. The net single premium for a joint and lastsurvivor annuity issued to lives aged $x$ and $y$ is

$$
\tilde{a}_{\overline{x y}}=\sum_{k=0}^{\infty} v_{k p_{\overline{x y}}}
$$

where ${ }_{k} \mathrm{p}_{\overline{x y}}=1-\mathrm{H}_{T}(k, k)$ is the conditional probability that at least one life survives an additional $k$ years. Here, the conditional distribution $\mathrm{H}_{T}$ is as defined in equation (3.6) with $a=0$. Because $\mathrm{H}_{T}$ is a function of the vector of parameters $\theta$, so are $k_{k} \bar{p}_{\overline{x y}}$ and $\bar{a}_{\overline{x y}}$. We will occasionally use the notation $a_{\overline{x y}}(\theta)$ to emphasize this dependence.

\subsection{Effects of Age and Interest}

In this subsection, we identify contract initiation ages $x$ and $y$ and interest environment $i$ where models of dependent mortality really matter. The approach is straightforward: we estimate the annuity value $\ddot{a}_{\overline{x y}}$ in equation (4.1) with and without assuming independence. The parameter estimates with and without independence are in Table 2 and were discussed in Subsection 3.2. We compare the annuity values by calculating the ratio of annuity values estimated without an independence assumption to those estimated with the independence assumption.

To assess the effects of contract initiation ages, Figure 4 presents a three-dimensional plot of the ratio of annuity values, by male $(x)$ and female (y) ages. The curve is roughly symmetric in $x$ and $y$, indicating that although the two marginal distributions are different they have approximately the same effect on the ratio. Further, there appears to be an interaction effect of $x$ and $y$ on the ratios. That is, the ratio is much smaller for large values of both $x$ and $y$ when compared to large values for either $x$ or for $y$.

To gain further understanding of this interaction effect, Figure 5 presents a multiple scatter plot of the ratio versus male ages, over several female ages. Here, for young female ages, we see that the ratio increases as male age increases. However, for older female ages, the ratio decreases as male age increases. 
Ratios that are less than one indicate that annuity values calculated assuming independence of lives are larger than those calculated without assuming independence. For this data set, it turns out that the average contract initiation age was approximately 65 and 63 for males and females, respectively. The average age as at December 31, 1993 was 72.5 and 69.6 for male and female lives, respectively. We interpret the higher ratios for younger ages to mean that the effect of assuming independence is smaller for premium determination compared to annuity reserve setting. This suggests that reserves for annuities already paid-up are larger than necessary.

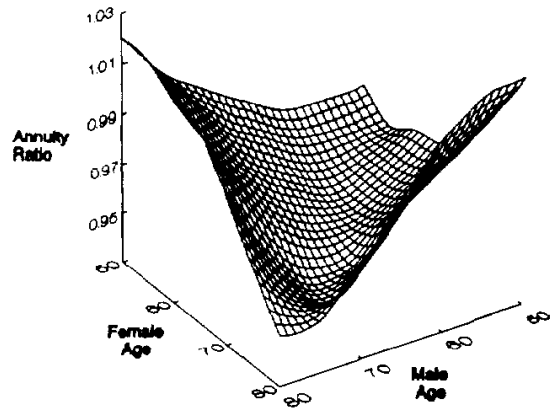

Figure 4. Three-dimensional plot of the ratio of dependent to independent annuity values, over several male and fermales ages. Here, five percent interest is assumed.

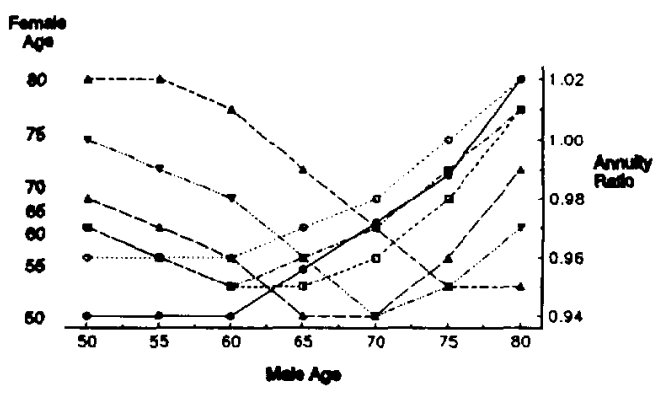

Figure 5. Multiple scatter plot of the ratio of dependent to independent annuity values to male age, over several females ages. Here, five percent interest is assurmed.

Our data set displays a strong relationship between $x$ and $y$. An examination of the data showed that the median age difference is 2.4 years, the middle fifty percent of the data is between 0.1 and 5.2 years, and the middle ninety percent is between -3.7 and 11.0 years. Because of this concentration, for brevity in our subsequent analyses, we present only the special case of $x=y$.

To assess the effects of interest, Figure 6 presents a three-dimensional plot of the ratio of annuity values, over several interest rates and ages. Here, the male age is assumed equal to the female age. This figure shows a quadratic effect of joint age that can also be observed in Figure 4. The effects of the interest rate $i$ seem to be linear.

To investigate these effects further, Figure 7 presents a multiple scatter plot of the ratio of annuity values to age, over several interest rates. This plot also demonstrates the quadratic effect of joint age and the linear effect of interest rates. From this plot, we see that the assumption of independence will matter more in times of low than high interest rates. 


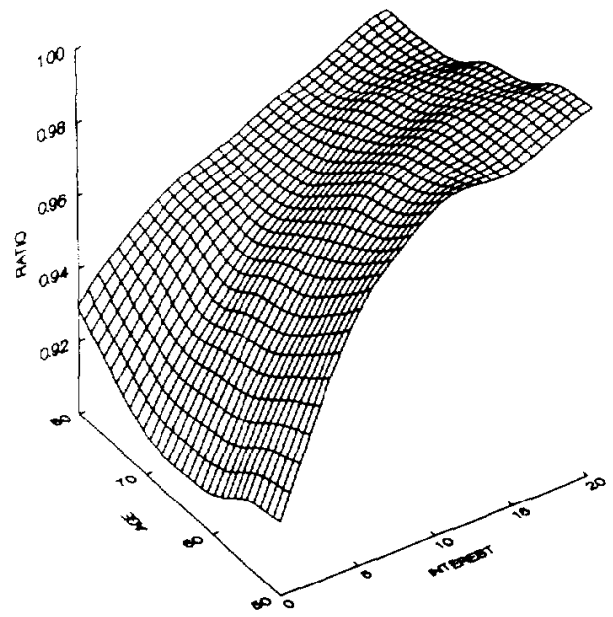

Figure 6. Three-dimensional plot of the ratio of dependent to independent annuity values, over several interest rates and ages. Here, equal annuitant ages are assumed.

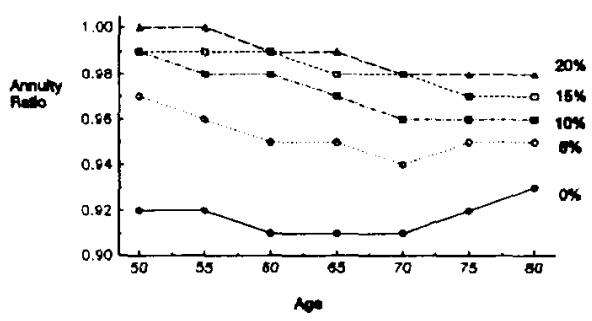

Figure 7. Multiple scatter plot of the ratio of dependent to independent annuity values to age, over several interest rates. Here, equal annuitant ages are assumed.

\subsection{Effects of Dependence on Other Annuities}

The joint and last survivor annuity is a special case of a broad class of joint-life annuities. In this subsection, we consider joint and $r$ annuities, where typically $r$ is two-thirds or one-half. For example, the joint and two-thirds annuities pay $\$ 1$ while both annuitants are alive and $\$ 2 / 3$ while one annuitant is alive. In the U.S., there may be a larger market for these annuities than the joint and last-survivor annuities that corresponds to $r=1$. This is because the Employee Retirement Income Security Act (ERISA) mandates that all qualified pension plans offer to qualified beneficiaries a joint and survivor annuity with $r$ at least fifty percent. For this purpose, in addition to the usual requirements, beneficiaries must be married to their current spouse for at least one year. Joint-life annuities, corresponding to $r=$ 0 , are not widely marketed.

Similar to equation (4.1), the net single premium for a joint and $r$ annuity can be expressed as

$$
\vec{a}_{x y}(r)=\sum_{k=0}^{\infty} \nu^{k}\left(r_{k} p_{x}+r_{k} p_{y}-(2 r-1) p_{x y}\right)
$$

where ${ }_{k} \mathrm{p}_{x}=1-\mathrm{H}_{T}(k, \infty)$ is the conditional probability that a life age $x$ survives an additional $k$ years, 
${ }_{k} \mathrm{p}_{y}=1-\mathrm{H}_{T}(\infty, k)$ is the conditional probability that a life age $y$ survives an additional $k$ years and ${ }_{k} p_{x y}$ $={ }_{k} \mathrm{p}_{x}+{ }_{k} \mathrm{p}_{y}-{ }_{k} \mathrm{p}_{\overline{x y}}=1-\mathrm{H}_{T}(k, \infty)-\mathrm{H}_{T}(\infty, k)+\mathrm{H}_{T}(k, k)$ is the conditional probability that both lives ages $x$ and $y$ survive an additional $k$ years.

Table 3 summarizes the effects of dependence on the reduced annuities. This tables shows that the reduction factor $r$ has little effect on the annuity ratios. As discussed above, we are primarily concerned with reduction factors $r=1 / 2,2 / 3$ and $I$ because these are the most widely marketed types of annuities.

\begin{tabular}{|c|c|c|c|c|c|c|}
\hline \multirow{2}{*}{ Age } & \multicolumn{6}{|c|}{$r$} \\
\hline & 0 & $1 / 4$ & $1 / 3$ & $1 / 2$ & $2 / 3$ & 1.0 \\
\hline 50 & 1.00 & 0.99 & 0.99 & 0.98 & 0.98 & 0.97 \\
\hline 55 & 1.00 & 0.98 & 0.98 & 0.98 & 0.97 & 0.96 \\
\hline 60 & 0.99 & 0.98 & 0.98 & 0.97 & 0.96 & 0.95 \\
\hline 65 & 0.98 & 0.97 & 0.97 & 0.96 & 0.96 & 0.95 \\
\hline 70 & 0.97 & 0.96 & 0.96 & 0.95 & 0.95 & 0.94 \\
\hline 75 & 0.94 & 0.94 & 0.94 & 0.94 & 0.94 & 0.94 \\
\hline 80 & 0.89 & 0.91 & 0.92 & 0.93 & 0.94 & 0.95 \\
\hline
\end{tabular}




\subsection{Annuity Standard Errors}

The annuity values calculated in Subsections 4.1 and 4.2 are based on the point estimate $\partial$ and thus depend on the sample. To measure the reliability of $\partial$, in Subsection 3.3 we discussed how the estimated variance-covariance matrix $V_{n}(\hat{\theta})$ could be used to derive parameter estimate standard errors. This subsection develops standard errors for $\vec{a}_{\overline{x y}}(\theta)$. These standard errors, together with the asymptotic normality, will allow us to provide confidence intervals for our joint and last-survivor annuity values.

The asymptotic normality of $\bar{a}_{\overline{x y}}(\hat{\theta})$ is based on the asymptotic normality of $\hat{\theta}$ and the so-called "delta-method" (see, for example, Serfling, 1980, Section 3.3). From Subsection 3.3, we have that

$$
n^{1 / 2}(\theta-\theta) \text { is } \operatorname{AN}\left(0, V_{n}(\theta)\right)
$$

where "AN $(\mathbf{0}, \mathbf{A})$ " means asymptotically normal with mean vector $\mathbf{0}$ and variance matrix $\mathbf{A}$. Define the gradient vector $\mathbf{G}(\theta)=\partial \vec{a}_{\overline{x y}}(\theta) / \partial \theta$. From the delta-method, we have

$$
n^{1 / 2}\left(\ddot{a} \overline{x y}(\theta)-a_{\overline{x y}}(\theta)\right) \text { is } \mathrm{AN}\left(0, \mathrm{G}(\theta)^{\prime} V_{n}(\theta) \mathrm{G}(\theta)\right)
$$

Thus, we may define the standard error of $a_{\overline{x y}}(\partial)$ to be

$$
\operatorname{se}\left(\mathrm{a}_{\overline{x y}}(\boldsymbol{\theta})\right)=\left(\mathbf{G}(\dot{\theta})^{\prime} V_{n}(\boldsymbol{\theta}) \mathbf{G}(\boldsymbol{\theta}) / n\right)^{1 / 2}
$$

From equation (4.3), we have that $\mathrm{d}_{\overline{x y}}(\hat{\theta}) \pm 1.96 \operatorname{se}\left(\mathrm{d}_{x y}(\hat{\theta})\right)$ provides an approximate $95 \%$ confidence interval for our annuity value $\frac{\alpha_{x y}}{(\theta)}$.

When computing the standard error, the most difficult component is the gradient vector. This is because, as noted in Subsection 3.3, the matrix $V_{n}(\partial)$ is an automatic output from standard function maximization routines. To compute the gradient vector, from equation (4.1), we have

$$
\mathbf{G}(\theta)=\frac{\partial}{\partial \theta}{ }_{x}(\theta)=\sum_{k=0}^{-} v^{k} \frac{\partial}{\partial \theta} k_{\overline{x y}}(\theta)=-\sum_{k=0}^{\infty} v^{k} \frac{\partial}{\partial \theta} H_{T}(k, k) .
$$

From equation (3.6) and the chain rule, we have 
$-\frac{\partial}{\partial \theta} \mathrm{H}_{T}(k, k)=(1-\mathrm{H}(x, \infty)-\mathbf{H}(\infty, y)+\mathrm{H}(x, y))^{-2}$

$$
\begin{gathered}
{\left[(\mathrm{H}(x+k, y+k)-\mathrm{H}(x, y+k)-\mathrm{H}(x+k, y)+\mathrm{H}(x, y)) \frac{\partial}{\partial \theta}(1-\mathrm{H}(x, \infty)-\mathrm{H}(\infty, y)+\mathrm{H}(x, y))\right.} \\
\left.-(1-\mathrm{H}(x, \infty)-\mathrm{H}(\infty, y)+\mathrm{H}(x, y)) \frac{\partial}{\partial \theta}(\mathrm{H}(x+k, y+k)-\mathrm{H}(x, y+k)-\mathrm{H}(x+k, y)+\mathrm{H}(x, y))\right] .
\end{gathered}
$$

Using equation (4.5), joint and last-survivor annuity standard errors were computed over several ages and interest rates. Table 4 presents the results for a five percent interest rate. The most important aspect of Table 4 is the magnitude of $\operatorname{se}\left(a_{\overline{x y}}(\theta)\right)$. To illustrate, consider our largest and smallest estimated annuity values which turn out to be $a_{50: 50}(\theta)=17.45$ and $a_{80: 80}(\theta)=9.65$. For the largest annuity values, the standard error represents a typical error that is $0.002 / 17.45=0.011$ percent of the annuity. For the smallest annuity value, the standard error represents a typical error that is $0.025 / 9.65=0.26$

\begin{tabular}{|c|c|c|c|c|c|c|c|}
\hline \multicolumn{8}{|c|}{$\begin{array}{l}\text { TABLE 4. Annuity Standard Errors by Male and Female Age } \\
\text { Five Percent Interest is Assumed. }\end{array}$} \\
\hline \multirow{2}{*}{$\begin{array}{c}\text { Male } \\
\text { Age }\end{array}$} & \multicolumn{7}{|c|}{ Female Age } \\
\hline & 50 & 55 & 60 & 65 & 70 & 76 & 80 \\
\hline 50 & 0.002 & 0.002 & 0.002 & 0.002 & 0.002 & 0.003 & 0.005 \\
\hline 55 & 0.002 & 0.002 & 0.003 & 0.002 & 0.002 & 0.003 & 0.004 \\
\hline 60 & 0.002 & 0.002 & 0.003 & 0.005 & 0.004 & 0.004 & 0.004 \\
\hline 65 & 0.002 & 0.002 & 0.003 & 0.006 & 0.008 & 0.008 & 0.006 \\
\hline 70 & 0.002 & 0.003 & 0.003 & 0.006 & 0.010 & 0.014 & 0.013 \\
\hline 75 & 0.002 & 0.003 & 0.004 & 0.006 & 0.009 & 0.016 & 0.023 \\
\hline 80 & 0.003 & 0.004 & 0.007 & 0.009 & 0.011 & 0.015 & 0.025 \\
\hline
\end{tabular}
percent. Thus, the standard errors indicate that the estimated annuity values are very accurate. 


\section{The Problem of Underreporting}

As discussed in Section 2, the data analyzed in this paper come from internal records of a large insurance company. Thus, in most cases the accuracy of the observed lifetimes depended on the reporting behavior of policyholders. This section investigates two instances where there may exist substantial measurement errors. The first involves underreporting of deaths within the guarantee period and the second involves underreporting of the first death. In each case, the approach is to reformulate the likelihood equation so that we are essentially re-estimating the Section 3 model using only subsets of our data. The subsets are chosen to circumvent the potential bias due to underreporting. Unfortunately, by using only subsets of the data, it turns out that we are unable to estimate the parameter values accurately when assessing potential underreporting of the first death. However, we do present the theoretical development of the likelihood equation to handle this type of underreporting.

\subsection{Underreporting Within the Guarantee Period}

Many policyholders elect a standard option that guarantees annuity payments will be made within a contractually specified period regardless of the mortality status of the annuitants. Because of the lack of financial incentives, there is concern that policyholders may not accurately report deaths that occur during the guarantee period. Further, it may be that mortality patterns for those electing a guarantee option may differ from those who do not. Of the 14,889 joint life contracts that we used for estimation, 10,011 contracts were at least partially guaranteed during the observation period.

To handle this potential bias, we re-estimated the model by disregarding mortality events within the guarantee period. This was done by censoring our lifetime data from the left at the expiration of the guarantee period. A consequence of this left censoring is that, for policies whose contract guarantee exceeded the observation period, there is no variability in the observed lifetimes and hence these policies were completely excluded from the likelihood calculations. Of the 14,889 contracts, 9,172 had guarantees that exceed the observation period.

To define the variables needed for the new likelihood function, let $c$ be the time since contract initiation of the guarantee period. Define $g=c-a$ to be the time from the beginning of the observation period to the end of the guarantee period (which may be negative). For contracts without a guarantee, we define $g=0$.

Our new likelihood is based on the right- and left-censored times at death 


$$
T_{j}^{* *}=\min \left(\max \left(T_{j}, g\right), b\right)
$$

and indicators of the type of censoring

$$
\begin{aligned}
& \delta_{j z}=\left\{\begin{array}{lll}
1 & \text { if } T_{j}^{* *}=8 & \text { (left-censored) } \\
b & \text { otherwise }
\end{array}\right. \\
& \delta_{j b}= \begin{cases}1 & \text { if } T_{j}^{* *}=b \\
0 & \text { otherwise }\end{cases}
\end{aligned}
$$

for $j=1,2$. Because contracts with guaranteed period exceeding the observation period are excluded from the likelihood function, we may assume $g<b$ without loss of generality.

The development of the guarantee period likelihood function is similar to that described in Subsection 3.3. Because it is more complex, the details are included in Appendix B. Using the guarantee likelihood is similar to the classic "select-and-ultimate" tables in life analysis, where the experience of policyholders during the select period is not used for calculating ultimate mortality rates.

Table 5 presents the estimation results for the new likelihood that accounts for the presence of the guarantee period. The parameter estimates from the guarantee period likelihood do not differ significantly from those of the full likelihood. As anticipated, the standard errors are larger for the guarantee period than for those of the full likelihood. This is due to the fact that we are using less information under the guarantee period censoring.

Table 5 does not suggest the presence of adverse selection by policyholders who elect the guarantee option. On the one hand, if there was an underreporting of deaths within the guarantee period, then we would expect the modal ages to decrease. On the other hand, if policyholders with poorer health elect guarantee options, then we would expect the modal ages to increase. Our guarantee likelihood estimates do not significantly differ from the full likelihood estimates, thus providing no conclusive evidence of the presence of adverse selection.

Table 5 also shows that our estimate of dependence has decreased to $\rho(\hat{\alpha})=\rho(-2.92)=0.44$. Although not statistically different from the full likelihood estimates, there may be some economic significance. Table 6 shows that this is not the case. The Table 6 ratios are approximately equal to the ratios discussed in Subsection 3.2. This again illustrates the highly nonlinear nature of the dependence parameter; large changes in $\alpha$ are needed to induce even small changes in our ratios of annuity values. 


\begin{tabular}{ccccc}
\hline \multicolumn{5}{c}{ TABLE 5. Guarantee Period Parameter Estimates } \\
\hline \multirow{4}{c}{ Full Likelihood } & \multicolumn{3}{c}{ Guarantere Period Likelihood } \\
Parameter & Estimate & Standard Error & Estimate & Standard Error \\
\hline$m_{1}$ & 85.82 & 0.26 & 84.78 & 0.40 \\
$\sigma_{1}$ & 9.98 & 0.40 & 9.58 & 0.49 \\
$m_{2}$ & 89.40 & 0.48 & 89.53 & 0.78 \\
$\sigma_{2}$ & 8.12 & 0.34 & 7.82 & 0.40 \\
$\alpha$ & -3.367 & 0.346 & -2.92 & 0.623 \\
\hline
\end{tabular}

\begin{tabular}{|c|c|c|c|c|c|c|c|}
\hline \multicolumn{8}{|c|}{$\begin{array}{l}\text { TABLE 6. Ratios of Dependent to Independent Joint and Last Annuity } \\
\text { Values Based on Guarantec Likelihood Estimates. } \\
\text { Five Percent Interest is Assumed. }\end{array}$} \\
\hline \multirow{2}{*}{$\begin{array}{c}\text { Male } \\
\text { Age }\end{array}$} & \multicolumn{7}{|c|}{ Female Age } \\
\hline & 50 & 55 & 60 & 65 & 70 & 75 & 80 \\
\hline so & 0.97 & 0.96 & 0.96 & 0.96 & 0.97 & 0.99 & 1.01 \\
\hline 55 & 0.97 & 0.96 & 0.95 & 0.95 & 0.96 & 0.97 & 1.00 \\
\hline 60 & 0.97 & 0.96 & 0.95 & 0.94 & 0.94 & 0.96 & 0.99 \\
\hline 65 & 0.98 & 0.97 & 0.96 & 0.94 & 0.93 & 0.94 & 0.97 \\
\hline 70 & 0.99 & 0.98 & 0.97 & 0.95 & 0.94 & 0.93 & 0.94 \\
\hline 75 & 1.01 & 1.00 & 0.99 & 0.98 & 0.96 & 0.93 & 0.92 \\
\hline 80 & 1.01 & 1.01 & 1.01 & 1.01 & 0.99 & 0.96 & 0.93 \\
\hline
\end{tabular}

\subsection{Underreporting of First Death}

For joint and last-survivor annuity policies, payments are made until the second, or last, annuitant dies. There is concern that policybolders might not report the death of only one annuitant, especially if there is no effect on the level of payment. For our data, of the 2,126 deaths $(=1,554$ males +572 females), 1,668 deaths left the other annuitant surviving. The other 458 deaths resulted in the cessation of payments on 229 contracts by the end of the observation period.

To handle this potential bias, we re-estimated the model by redefining "failure" to be the time of second death, that is, cessation of the policy. This approach treats the 1,668 single deaths as policies that "survive" the observation period. 
Our new likelihood is based on the time of second death

$$
\left.T^{*}=\max \left(T_{1}^{*}, T_{2}^{*}\right)=\max \left(\min \left(T_{1}, b\right), \min \left(T_{2}, b\right)\right)=\min \left(\max \left(T_{1}, T_{2}\right), b\right)\right)
$$

and the indicator of censoring, $\delta^{*}$, which is one if $T^{*}=b$ and zero otherwise. Similar to Subsection 3.3, the likelihood function is based on two cases.

1. If the second death is uncensored, then we may assume $t<b$. In this case, we have $\delta^{*}=0$ and

$\operatorname{Prob}\left(T^{*}<t \mid T_{1}>0, T_{2}>0\right)=\operatorname{Prob}\left(T_{1}<t, T_{2}<t \mid T_{1}>0, T_{2}>0\right)=\mathrm{H}_{T^{(t}}(t)$.

Using the chain rule, we have $\partial \mathrm{H}(t, t) / \partial t=\mathrm{H}_{1}(t, t)+\mathrm{H}_{2}(t, t)$. Thus, the contribution to the likelihood function is:

$$
\frac{\partial \mathrm{H}_{\mathrm{T}}(t, t)}{\partial t}=\frac{\mathrm{H}_{1}(x+a+t, y+a+t)+\mathrm{H}_{2}(x+a+t, y+a+t)-\mathrm{H}_{2}(x+a, y+a+t)-\mathrm{H}_{1}(x+a+t, y+a)}{1-\mathrm{H}(x+a, \infty)-\mathrm{H}(\infty, y+a)+\mathrm{H}(x+a, y+a)} .
$$

2. If the second death is censored, then we may assume $t \geq b$. In this case, we have $\delta^{*}=1$ and

$$
\operatorname{Prob}\left(T^{*}=b \mid T_{1}>0, T_{2}>0\right)=1-\mathrm{H}_{T}(b, b)
$$

Combining equations (5.1) and (5.2), we can express the logarithm of the likelihood function for a single observation as

$$
\ln L\left(x, y, t, \delta^{*}, a, b\right)
$$

$$
\begin{gathered}
=\left(1-\delta^{\circ}\right) \ln \left(\mathrm{H}_{1}(x+a+t, y+a+t)+\mathrm{H}_{2}\left(x+a+t_{3} y+a+t\right)-\mathrm{H}_{2}(x+a, y+a+t)-\mathrm{H}_{1}(x+a+t, y+a)\right) \\
+\delta^{*} \ln (1-\mathrm{H}(x+a, \infty)-\mathrm{H}(\infty, y+a)+\mathrm{H}(x+a, y+a+b)+\mathrm{H}(x+a+b, y+a)-\mathrm{H}(x+a+b, y+a+b)) \\
-\ln (1-\mathrm{H}(x+a, \infty)-\mathrm{H}(\infty, y+a)+\mathrm{H}(x+a, y+a)) .
\end{gathered}
$$

The log-likelihood for the data can be calculated using the equation (5.3) expression in equation (3.12). Maximizing this log-likelihood function yields parameter estimates and standard errors, as in Subsection 3.3. 
Although this approach is technically sound, the maximum likel ihood method yielded unreliable parameter estimates using our data. Despite having nearly fifteen thousand contracts (and almost thirty thousand lives) available for estimation, with this reduced data set we had only 229 deaths. Intuitively, most of the parameter information comes from the deaths, and we are attempting to estimate five parameters (in addition to the variance-covariance matrix). Thus, larger data sets, or longer observation periods, may be required to implement the method of this subsection. This is interesting because generally data analysts do not consider fifteen thousand observations to a data set to be too small to employ likelihood methods.

\section{Alternative Models of Dependence}

This section investigates the robustness of the choice of Gompertz marginals and Frank's family of copulas by presenting some alternative choices.

\subsection{Weibull Marginal Distribution}

The Weibull distribution function can be expressed as:

$$
F(x)=1-\exp \left(-(x / m)^{m^{\prime} /}\right)
$$

where $m$ and $\sigma$ are location and scale parameters. The mode of this distribution is $m(1-\sigma / m)^{\sigma / m}$ which is approximately $0.98 m$ for $m=80$ and $\sigma=10$. Thus, because our estimated values of $m$ and $\sigma$ turn out to be close to 80 and 10 , respectively, we may interpret $m$ to be an approximate mode for this distribution, similar to the Gompertz. A more traditional expression for the Weibull is

$$
F(x)=1-\exp \left(-B x^{c}\right)
$$

which is equivalent with the transformations $B=m^{-m / \sigma}$ and $c=m / \sigma$. Similarly to the case of the Gompertz distribution, we find the parameterization in equation (6.1) to be more convenient for computational purposes.

Appendix D presents the Weibull parameter estimates and annuity ratios. Similar to Section 3, we used maximum likelihood to estimate the parameter values. The annuity ratios were computed following the same format that was described in Section 4.

As suggested by Figures 1 and 2, the annuity ratios from the Weibull and Gompertz marginals are very similar. In most cases, the ratios differ by 0.01 or less. This suggests that our ratio values are 
not sensitive to the choice of marginal distributions. It is an interesting area of future research to measure the extent of the dependence of the ratios on the underlying marginal distributions.

\subsection{Shock Models of Dependence}

In this section, we investigate the effects of the choice of the copula by considering an alternative family, the "common shock" models. As pointed out by Panjer (1994), the primary advantages of the common shock models are that they are easy to interpret and are computationally convenient.

To define this bivariate distribution, we begin with independent age-at-death random variables $X$ and $Y$. As in Subsection 3.1, we denote their marginal distribution functions by $F_{j}$ so that $F_{1}(x)=$ $\operatorname{Prob}(X \leq x)$ and $F_{2}(y)=\operatorname{Prob}(Y \leq y)$. We assume there exists an independent exponential random variable $Z$ with parameter $\lambda$, that is, $\operatorname{Prob}(Z \leq t)=1-\mathrm{e}^{-\lambda s}$. The bivariate time-until-death random vector is $(T(x), T(y))$, where $T(x)=\min (X-x-a, Z)$ and $T(y)=\min (Y-y-a, Z)$. With this, we interpret $Z$ to be a "shock" that is common to both lives. Our new underlying lifetime random variables are $X_{c}=$ $T(x)+x+a=\min (X, Z+x+a)$ and $Y_{c}=T(y)+y+a=\min (Y, Z+y+a)$.

Under these assumptions, it is straightforward to compute the bivariate distribution. The survival distribution can be expressed as, for $t_{1}, t_{2} \geq 0$,

$$
\begin{gathered}
\operatorname{Prob}\left(X_{c}>x+a+t_{1}, Y_{c}>y+a+t_{2}\right)=\operatorname{Prob}\left(\min (X, Z+x+a)>x+a+t_{1}, \min (Y, Z+y+a)>y+a+t_{2}\right) \\
=\operatorname{Prob}\left(Z>\max \left(t_{1}, t_{2}\right)\right) \operatorname{Prob}\left(X>x+a+t_{1}\right) \operatorname{Prob}\left(Y>y+a+t_{2}\right) \\
=\exp \left(-\lambda \max \left(t_{1}, t_{2}\right)\right)\left(1-\mathrm{F}_{1}\left(x+a+t_{1}\right)\right)\left(1-\mathrm{F}_{2}\left(y+a+t_{2}\right)\right) .
\end{gathered}
$$

Thus, the bivariate distribution function, for $t_{1}, t_{2} \geq 0$, is

$$
\begin{aligned}
H\left(x+a+t_{1}, y+a+t_{2}\right)= & \operatorname{Prob}\left(X_{c} \leq x+a+t_{1}, Y_{c} \leq y+a+t_{2}\right) \\
=1 & \exp \left(-\lambda t_{1}\right)\left(1-\mathrm{F}_{1}\left(x+a+t_{1}\right)\right) \\
& \quad-\exp \left(-\lambda t_{2}\right)\left(1-\mathrm{F}_{2}\left(y+a+t_{2}\right)\right)+\exp \left(-\lambda \max \left(t_{1}, b\right)\right)\left(1-\mathrm{F}_{1}\left(x+a+t_{1}\right)\right)\left(1-\mathrm{F}_{2}\left(y+a+t_{2}\right)\right) .
\end{aligned}
$$

From equation (6.3), we note that $\mathbf{H}(x+a+t, \infty)=1-\mathrm{e}^{-\lambda t}\left(1-\mathrm{F}_{1}(x+a+t)\right) \neq \mathrm{F}_{1}(x+a+t)$. Thus, unlike the case of the copula bivariate function, the marginal common shock distributions are a function of the dependence parameter $\lambda$.

Parameters were estimated using the bivariate distribution function in equation (6.3) and the method of maximum likelihood similar to the discussion in Subsection 3.3; the only difference is the 
likel ihood of a common shock. This is given by the instantaneous probability:

$$
\frac{\partial}{\partial t} \operatorname{Prob}(T(x)=T(y) \leq t)=\lambda \mathrm{e}^{-\lambda}\left(1-\mathrm{F}_{1}(x+a+t)\right)\left(1-\mathrm{F}_{2}(y+a+t)\right)
$$

Table 7 presents the parameter estimates with the associated standard errors. Here we find that the common shock location and scale parameters are close to the corresponding univariate estimates. All are within one standard error except $m_{1}$ that is only $1.3(=(86.66-86.38) / 0.27)$ standard errors away. The measure of dependence, $\hat{\lambda}$, is more than five standard errors from zero, indicating strong statistical dependence. Although not significant, we note that the location estimates are higher under the bivariate distribution than the univariate. Recall from Table 2 that the location estimates were significantly lower under the Gompertz/Frank model than the univariate models.

\begin{tabular}{|c|c|c|c|c|}
\hline \multirow[b]{2}{*}{ Parameter } & \multicolumn{2}{|c|}{ Bivariate Distribution } & \multicolumn{2}{|c|}{ Univariate Distributions } \\
\hline & Estimate & Standard Error & Estimate & Standard Error \\
\hline$m_{1}$ & 86.66 & 0.27 & 86.38 & 0.26 \\
\hline$\sigma_{1}$ & 9.89 & 0.37 & 9.83 & 0.37 \\
\hline$m_{2}$ & 92.69 & 0.64 & 92.17 & 0.59 \\
\hline$\sigma_{2}$ & 8.09 & 0.40 & 8.11 & 0.38 \\
\hline$\lambda$ & 0.00054 & 0.00010 & No Applicable & Not Applicable \\
\hline
\end{tabular}

The common shock model is intuitively appealing because bivariate conditional probabilities can easily be related to the marginals. To illustrate, recall from Section 4 that ${ }_{k} p_{\overline{x y}}=1-\mathrm{H}_{T}(k, k)$ is the conditional probability that at least one life survives an additional $k$ years. Using equations (6.2) and (6.3), straight-forward calculations show that

$$
{ }_{k} p_{\overline{x y}}={ }_{k} p_{x}+{ }_{k} p_{y}-e^{\lambda t} p_{x} p_{y} .
$$

Here, ${ }_{k} \mathrm{p}_{x}=(1-\mathrm{H}(x+k, \infty)) /(1-\mathrm{H}(x, \infty))=\mathrm{e}^{-\lambda k_{(1}}\left(1-\mathrm{F}_{1}(x+k)\right) /\left(1-\mathrm{F}_{1}(x)\right)$ is conditional probability that a life aged $x$ survives an additional $k$ years and similarly for ${ }_{k} p_{y}$. Thus, for example, we may express our joint and last-survivor annuity as 


$$
\ddot{a} \overline{x y}=\sum_{k=0}^{\infty} v^{k}\left({ }_{k} \mathrm{p}_{x}+{ }_{k p_{y}}-\mathrm{e}^{\lambda k}{ }_{k \mathrm{p}_{x} \mathrm{p}_{y}}\right)
$$

An intuitively appealing feature of the common shock model is that the dependence parameter, $\lambda$, can be absorbed into the interest parameter, as follows. Define the pseudo conditional probabilities ${ }_{k} \mathrm{p}_{x}^{*}$ $=\left(1-F_{1}(x+k)\right) /\left(1-F_{1}(x)\right)=e^{\lambda k}{ }_{k} p_{x}$ and similarly for ${ }_{k} p_{y}^{*}$. Using equations (6.4) and (6.5), we have

$$
\begin{aligned}
& \mathrm{a}_{\overline{x y}}=\sum_{k=0}^{-} \mathrm{e}^{-(\delta+\lambda) k}\left({ }_{k} \mathrm{p}_{x}^{*}+{ }_{k} \mathrm{p}_{y}^{*}-{ }_{k} \mathrm{p}_{x k}^{*} \mathrm{p}_{y}^{*}\right) \\
& =I_{\tilde{\mathrm{a}}}{ }_{\overline{x y}} @(\delta+\lambda) .
\end{aligned}
$$

Here, $\delta=\ln (1+i)$ is the so-called "force of interest, " the symbol ${ }_{\mathfrak{a}_{\bar{x}}}$ means calculate the annuity assuming independence using ${ }_{k} \mathrm{p}_{x}^{*}$ and ${ }_{k} \mathrm{p}_{y}^{*}$, and the notation "@ $(\delta+\lambda)^{\prime \prime}$ means at force of interest $\delta+\lambda$. Because ${ }_{k} p_{x}^{*}$ and ${ }_{k} p_{y}^{*}$ do not depend on $\lambda$, equation (6.6) shows that the joint and last-survivor annuity is a decreasing function of $\lambda$. In other words, the greater is the dependency, the smaller is the joint and lastsurvivor annuity.

To assess the real impact of dependency, Table 8 compares annuity values calculated under the common shock model to those calculated under independence. Unlike our copula models, Table 8 shows that annuity values are higher under the common shock model for most age combinations. This is interesting because, from equations (6.5) and (6.6), we would expect annuity ratios less than one. On one hand, the increase in $\lambda$ (from 0 to 0.00054 ) produces only a small decrease in annuity values. On the other hand, the larger location parameters mean that the individual forces of mortality were lower under the case of dependence. Lower forces of mortality resulted in larger annuity values. 


\begin{tabular}{|c|c|c|c|c|c|c|c|}
\hline \multirow{2}{*}{$\begin{array}{l}\text { Male } \\
\text { Age }\end{array}$} & \multicolumn{7}{|c|}{ Female Age } \\
\hline & 50 & 55 & 60 & 65 & 70 & 75 & 80 \\
\hline 50 & 1.00 & 1.00 & 1.00 & 1.00 & 1.00 & 1.00 & 1.00 \\
\hline 55 & 1.00 & 1.00 & 1.00 & 1.00 & 1.00 & 1.00 & 1.00 \\
\hline 60 & 1.00 & 1.00 & 1.00 & 1.00 & 1.00 & 1.00 & 1.00 \\
\hline 65 & 1.00 & 1.00 & 1.00 & 1.00 & 1.00 & 1.00 & 1.00 \\
\hline 70 & 1.00 & 1.00 & 1.00 & 1.00 & 1.01 & 1.01 & 1.01 \\
\hline 75 & 1.00 & 1.00 & 1.00 & $1 . \infty$ & 1.01 & 1.01 & 1.01 \\
\hline 80 & 1.00 & 1.00 & 1.00 & 1.01 & 1.01 & 1.01 & 1.01 \\
\hline
\end{tabular}

Thus, despite the computational simplicity, the common shock model does not seem to provide the same pleasing intuitive results as the copula model. Further, the common shock model does not seem to fit the data as well as the Gompertz/Frank model. When estimating the models, the log-likelihood associated with the Gompertz/Frank model, $-9,977$, was larger than the log-likelihood associated with the common shock model, $-10,078$. The two models are not hierarchical and thus traditional likelihood ratio tests are not applicable. However, this does provide additional evidence that the Frank/Gompertz model provides a better fit to the data.

Finally, we note that the common shock does not seem to take in account all the dependencies that we observe in the data. In our analysis, we found that, of the 229 pairs of deaths within our observation period, 29 occurred with one day and hence were "simultaneous." The data also revealed proximity of other deaths:

\begin{tabular}{cc}
\hline Period of Days & $\begin{array}{c}\text { Number of Pairs of Deaths } \\
\text { Within the Period of Days }\end{array}$ \\
\hline 1 Day & 29 \\
5 Days & 63 \\
10 Days & 70 \\
20 Days & 85 \\
30 Days & 86 \\
\hline
\end{tabular}


Thus, there appears to be some dependency of lives that the common shock model does not detect. Of course, one can always alter the definition of the time scale to re-define what "simultaneous" means. An advantage of the copula models is that this is not necessary because the dependency is assessed in a smooth fashion.

\section{Concluding Remarks}

In this article, we discussed methods for estimating the probability of joint survival using insurance data. Although our focus has been on annuity valuation, our methods can easily be applied to other types of insurance products. For example, Bragg (1994) discussed the growing importance of lastsurvivor, or "second-to-die," life insurance.

Throughout the article, our illustrations focused on valuing level annuities using fixed interest rates. However, with additional complexity, the methods of this paper can also be applied to variable products. They can also be used for valuing level annuities using a model from financial economics. This is because economic models assume that probabilities are exogenous inputs into an economic system. Thus, our dependent mortality models could be used to determine probabilities that are inputs to an economic model.

Because of the heavy truncation and censoring of our data, our models of the bivariate distribution are completely parametric. Recently, Maguluri (1993) has provided some theoretical results on the efficiency of using a parametric family for the copula, such as Frank's family, with nonparametric distributions for the marginals, such as Kaplan-Meier. It would be interesting to fit data using a parametric copula and standard insurance industry tables, such as the 1983 Individual Annuity Table. We leave this as an area for future research. 


\section{References}

American Council of Life Insurance, 1994, 1994 Life Insurance Fact Book, 1001 Pennsylvania Avenue, N.W., Washington, D.C. 20004-2599.

Bowers, N.L., Gerber, H.U., Hickman, J.C., Jones, D.A., Nesbitt, C.J., 1986, Actuarial Mathematics, Society of Actuaries, Schaumburg, IL.

Bragg, J. 1994, The Last-Survivor Phenomenon, The Actuary, January, page 5.

Carriere, J., 1994, An Investigation of the Gompertz Law of Mortality. ARCH, 1994.2.

Cox D. R. and Oakes, D., 1990, Analysis of Survival Data, New York, N. Y.: Chapman and Hall.

Elandt-Johnson, R.C. and Johnson, N.L., 1980, Survival Models and Data Analysis. New York, N.Y.: John Wiley \& Sons, Inc.

Frank, M.J., 1979, On the Simultaneous Associativity of $F(x, y)$ and $x+y-F(x, y)$, Aequationes Math. 19, 194-226.

Genest, C., 1987, Frank's Family of Bivariate Distributions, Biometrika 74, 549-555.

Genest, C. and McKay, J., 1986, The Joy of Copulas: Bivariate Distributions with Uniform Marginals, American Statistician 40, 280-283.

Hougaard, P., Garvald, B. and Holm, N. V., 1992, Measuring the Similarities Between the Lifetimes of Adult Danish Twins Born Between 1881-1930, Journal of the American Statistical Association 87, 17-24.

Jagger, C. and Sutton, C.J., 1991, Death after Marital Bereavement - Is the Risk Increased?, Statistics in Medicine 10, 395-404.

Maguluri, G., 1993, Semiparametric Estimation of Association in a Bivariate Survival Function, Annals of Statistics 21, 1648-1662.

Marshall, A.W. and Olkin, I., 1967, A Multivariate Exponential Distribution, Journal of the American Statistical Association 62, 30-44.

Marshall, A.W. and Olkin, I., 1988, Families of Multivariate Distributions, Joumal of the American Statistical Association 83, 834-841.

Nelsen, R.B., 1986, Properties of a One-Parameter Family of Distributions with Specified Marginals, Communications in Statistics - Theory and Methods A 15, 3277-3285.

Oakes, D., 1989, Bivariate Survival Models Induced by Frailties, Journal of the American Statistical Association 84, 487-493.

Panjer, H., 1994, Second-to-Die with Possibility of Simultaneous Death, Product Development News, Issue 36 (June), Society of Actuaries, Schaumburg, IL.

Parkes, C.M., Benjamin, B. and Fitzgerald, R.O., 1969, Broken Heart: A Statistical Study of Increased Mortality among Widowers, British Medical Journal i, 740-743.

Pollard, J. H. and Volkovics, E.J., 1993, The Gompertz Distribution and its Applications. Genus.

Pruitt, R.C., 1993, Identifiability of Bivariate Survival Curves from Censored Data, Journal of the American Staristical Association 88, 573-579.

Serfling, R. (1980), Approximation Theorems in Mathematical Statistics, John Wiley and Sons, New York.

Sklar, A., 1959, Fonctions de répartitions à n dimensions et leurs marges. Inst. Statist. Univ. Paris Publ. 8, 229-231.

Ward, A. 1976, Mortality of Bereavement, British Medical Journal i, 700-702. 


\section{APPENDIX A}

\section{Derivation of Results Needed to Evaluate the Likelihood \\ Using Frank's Copula and Gompertz Marginals}

In this appendix, we derive the results needed to evaluate the log-likelihood in (3.12), or in particular (3.11), in the case where we assume the Frank's copula function as given in (3.2) and "Gompertz" marginals as given in (3.4).

Let us consider the bivariate age-at-death random vector $(X, Y)$ whose distribution function is given in equation (3.1) and where the copula is given in equation (3.2). Deriving the first partial derivatives of $C$, we have:

$$
\begin{aligned}
& C_{1}(u, v)=\frac{\partial}{\partial u} C(u, v)=\frac{e^{\alpha u}\left(e^{\alpha v}-1\right)}{e^{\alpha}-1+\left(e^{\alpha u}-1\right)\left(e^{\alpha v}-1\right)} \\
& \text { and } \\
& C_{2}(u, v)=\frac{\partial}{\partial v} C(u, v)=\frac{e^{\alpha v}\left(e^{\alpha u}-1\right)}{e^{\alpha}-1+\left(e^{\alpha u}-1\right)\left(e^{\alpha v}-1\right)}
\end{aligned}
$$

The second partial derivative of $C$ is given as:

$$
C_{12}(u, v)=\frac{\partial^{2}}{\partial u \partial v} C(u, v)=\frac{\alpha\left(e^{\alpha}-1\right) e^{\alpha(u+\sigma)}}{\left[\left(e^{\alpha}-1\right)+\left(e^{\alpha u}-1\right)\left(e^{\alpha u}-1\right)\right]^{2}} .
$$

We denote the density functions of $X$ and $Y$ as $f_{1}$ and $f_{2}$, respectively. In other words, we have:

$$
f_{1}(x)=\frac{\partial}{\partial x} F_{1}(x) \text { and } f_{2}(y)=\frac{\partial}{\partial y} F_{2}(y)
$$

Using the chain rule of differentiation, we then have the first and second partial derivatives of the distribution function $H(x, y)$ :

$$
\begin{gathered}
H_{1}(x, y)=\frac{\partial}{\partial x} H(x, y)=f_{1}(x) C_{1}\left(F_{1}(x), F_{2}(y)\right) \\
\text { and } \\
H_{2}(x, y)=\frac{\partial}{\partial y} H(x, y)=f_{2}(y) C_{2}\left(F_{1}(x), F_{2}(y)\right) \\
\text { and } \\
h(x, y)=\frac{\partial^{2}}{\partial x \partial y} H(x, y)=f_{1}(x) f_{2}(y) C_{12}\left(F_{1}(x), F_{2}(y)\right) .
\end{gathered}
$$


If we suppose that the marginals follow Gompertz distribution as in equation (2.1), then we have the following density functions:

$$
f_{i}(x)=\frac{1}{\sigma_{i}} e^{\left(x-m_{1} / j \sigma_{i}\right.} \exp \left[e^{m_{i /} / \sigma_{i}}\left(1-e^{y / \sigma_{1}}\right)\right], \mathrm{j}=1,2
$$

Equations (A.1) to (A.3) and (A.7) above are then used to evaluate (A.4) to (A.6). Equations (A.4), (A.5) and (A.6) are used in maximizing the log-likelihood as expressed in (3.11).

Note that the parameter $\alpha$ is not necessarily a standard measure of association. However, we can express the more familiar Spearman's correlation coefficient as a function of $\alpha$ as follows:

$$
\rho(\alpha)=12 \int_{0}^{1} \int_{0}^{1} C(u, v) d u d v-3
$$

If $C$ is the Frank's copula, then we have $\rho(\alpha)$ as expressed in (3.3). 


\section{APPENDIX B \\ Development of the Likelihood Equation for Underreporting of Death Within the Guarantee Period}

To determine the conditional distribution of $\left(T_{1}^{*}, T_{2}^{* *}\right)$, we consider nine cases of $\left(t_{1}, t_{2}\right)$. The general expression for the conditional distribution function for the guaranteed case is:

$$
\begin{aligned}
H_{g}\left(t_{1}, t_{2}\right) & =\operatorname{Prob}\left(T_{1}^{*} \leq t_{1}, T_{2}^{* *} \leq t_{2} \mid T_{1}>0, T_{2}>0\right) \\
& =\operatorname{Prob}\left(\min \left(\max \left(T_{1}, g\right), b\right) \leq t_{1}, \min \left(\max \left(T_{2}, g\right), b\right) \leq t_{2} \mid T_{1}>0, T_{2}>0\right) .
\end{aligned}
$$

Case 1. If both lifetimes are right-censored, then we have $t_{1} \geq b, t_{2} \geq b$. Hence, we have $\delta_{1 g}=\delta_{2 g}=0, \delta_{1 b}=\delta_{2 b}=1$ and

$$
\begin{aligned}
H_{s}\left(t_{1}, t_{2}\right) & =\operatorname{Prob}\left(T_{1}^{* *}=b, T_{2}^{* *}=b \mid T_{1}>0, T_{2}>0\right) \\
& =\operatorname{Prob}\left(T_{1} \geq b, T_{2} \geq b \mid T_{1}>0, T_{2}>0\right) \\
& =1-H_{T}(\infty, b)-H_{T}(b, \infty)+H_{T}(b, b) .
\end{aligned}
$$

Case 2. If the first lifetime is uncensored and the second is right-censored, then we have $g<t_{1}<b, t_{2} \geq b$. Hence, we have $\delta_{1 g}=\delta_{2 g}=\delta_{1 b}=0, \delta_{2 b}=1$ and

$$
H_{g}\left(t_{1}, t_{2}\right)=\operatorname{Prob}\left(T_{1} \leq t_{1}, T_{2} \geq b \mid T_{1}>0, T_{2}>0\right)=H_{\mathrm{T}}\left(t_{1}, \infty\right)-H_{T}\left(t_{1}, b\right) .
$$

Case 3. If the first lifetime is left-censored and the second is right-censored, then we have $t_{1} \leq g, t_{2} \geq b$ Hence, we have $\delta_{1 g}=\delta_{2 b}=1, \delta_{1 b}=\delta_{2 g}=0$ and

$$
H_{g}\left(t_{1}, t_{2}\right)=H_{T}(g, \infty)-H_{T}(g, b) \text {. }
$$

Case 4. If the first lifetime is right-censored and the second is uncensored, then we have $t_{1} \geq b, g<t_{2}<b$. Hence, we have $\delta_{1 b}=1, \delta_{1 g}=\delta_{2 g}=\delta_{2 b}=0$ and

$$
H_{g}\left(t_{1}, t_{2}\right)=H_{T}\left(\infty, t_{2}\right)-H_{T}\left(b, t_{2}\right)
$$

Case 5. If both lifetimes are uncensored, then we have $g<t_{1}<b, g<t_{2}<b$. Hence we have $\delta_{1 g}=\delta_{1 b}=0, \delta_{2 g}=\delta_{2 b}=0$ and

$$
H_{g}\left(t_{1}, t_{2}\right)=H_{T}\left(t_{1}, t_{2}\right)
$$

Case 6. If the first lifetime is left-censored and the second lifetime is uncensored, then we have $t_{1} \leq g, \mathrm{~g}<t_{2}<b$. Hence, we have $\delta_{1 g}=1, \delta_{2 g}=\delta_{1 \mathrm{~b}}=\delta_{2 b}=0$ and

$$
H_{g}\left(t_{1}, t_{2}\right)=\operatorname{Prob}\left(T_{1} \leq g, T_{2} \leq t_{2}\right)=H_{T}\left(g, t_{2}\right) \text {. }
$$


Case 7. If the first lifetime is right-censored and the second is left-censored, then we have $t_{1} \geq b, t_{2} \leq g$. Hence, we have $\delta_{1 b}=\delta_{2 q}=1, \delta_{1 x}=\delta_{2 b}=0$ and

$$
H_{y}\left(t_{1}, t_{2}\right)=\operatorname{Prob}\left(T_{1} \geq b, T_{2} \leq g\right)=H_{T}(\infty, g)-H_{T}(b, g) .
$$

Case 8 . If the first lifetime is uncensored and the second is left-censored, then we have $g<t_{1}<b, t_{2} \leq g$. Hence, we have $\delta_{1 g}=\delta_{1 b}=\delta_{2 b}=0, \delta_{2 g}=1$ and

$$
H_{g}\left(t_{1}, t_{2}\right)=H_{T}\left(t_{1}, g\right)
$$

Case 9. Finally, if both lifetimes are left-censored, we have $t_{1} \leq g$ and $t_{2} \leq g$. Hence, we have $\delta_{1 g}=\delta_{2 g}=1, \delta_{1 b}=\delta_{2 b}=0$ and

$$
H_{g}\left(t_{1}, t_{2}\right)=H_{T}(g, g)
$$

Recall from equations (3.7)-(3.9) that:

$$
\begin{aligned}
& \frac{\partial H_{T}\left(t_{1}, t_{2}\right)}{\partial t_{1}}=\frac{H_{1}\left(x+a+t_{1}, y+a+t_{2}\right)-H_{1}\left(x+a+t_{1}, y+a\right)}{1-H(x+a, \infty)-H(\infty, y+a)+H(x+a, y+a)}, \\
& \frac{\partial H_{T}\left(t_{1}, t_{2}\right)}{\partial t_{2}}=\frac{H_{2}\left(x+a+t_{1}, y+a+t_{2}\right)-H_{2}\left(x+a, y+a+t_{2}\right)}{1-H(x+a, \infty)-H(\infty, y+a)+H(x+a, y+a)}, \\
& \frac{\partial^{2} H_{T}\left(t_{1}, t_{2}\right)}{\partial t_{1} \partial t_{2}}=\frac{\text { and }}{1-H(x+a, \infty)-H(\infty, y+a)+H(x+a, y+a)} .
\end{aligned}
$$

Combining equations (B.2) to (B.10), we then have the contribution of a single observation to the log-likelihood as follows:

$$
\begin{aligned}
\log & L_{g}\left(x, y, a, b, t_{1}, t_{2}, \delta_{1 g}, \delta_{2 g}, \delta_{1 b}, \delta_{2 b}, g\right) \\
= & \delta_{1 b} \delta_{2 b} \log [1-H(\infty, y+a+b)-H(x+a+b, \infty)+H(x+a+b, y+a+b)] \\
& +\left(1-\delta_{1 g}-\delta_{1 b}\right) \delta_{2 b} \log \left[H_{1}\left(x+a+t_{1}, \infty\right)-H_{1}\left(x+a+t_{1}, y+a+b\right)\right] \\
& +\delta_{1 g} \delta_{2 b} \log [H(x+a+g, \infty)-H(x+a, \infty)-H(x+a+g, y+a+b)+H(x+a, y+a+b)] \\
& +\delta_{1 b}\left(1-\delta_{2 g}-\delta_{2 b}\right) \log \left[H_{2}\left(\infty, y+a+t_{2}\right)-H_{2}\left(x+a+b, y+a+t_{2}\right)\right] \\
& +\left(1-\delta_{1 b}-\delta_{1 g}\right)\left(1-\delta_{2 g}-\delta_{2 b}\right) \log \left[h\left(x+a+t_{1}, y+a+t_{2}\right)\right] \\
& +\delta_{1 g}\left(1-\delta_{2 g}-\delta_{2 b}\right) \log \left[H_{2}\left(x+a+g, y+a+t_{2}\right)-H_{2}\left(x+a, y+a+t_{2}\right)\right] \\
& +\delta_{1 b} \delta_{2 g} \log [H(\infty, y+a+g)-H(\infty, y+a)-H(x+a+b, y+a+g)+H(x+a+b, y+a)] \\
& +\left(1-\delta_{1 b}-\delta_{1 k}\right) \delta_{2 g} \log \left[H_{1}\left(x+a+t_{1}, y+a+g\right)-H_{1}\left(x+a+t_{1}, y+a\right)\right] \\
& +\delta_{1 g} \delta_{2 g} \log [H(x+a+g, y+a+g)-H(x+a+g, y+a)-H(x+a, y+a+g)+H(x+a, y+a)] \\
& -\log [1-H(x+a, \infty)-H(\infty, y+a)+H(x+a, y+a)] .
\end{aligned}
$$




\section{APPENDIX C \\ Calculation of the Gradient of $\ddot{a}_{\overline{x y}}(\theta)$}

Let $\theta=\left(m_{1}, m_{2}, \sigma_{1}, \sigma_{2}, \alpha\right)^{\prime}=\left(\theta_{1}, \theta_{2}, \theta_{3}, \theta_{4}, \theta_{5}\right)^{\prime}$. Thus, from equation $(4.5)$ for $i=1,2, \ldots 5$, we have:

$$
\begin{aligned}
& \frac{\partial}{\partial \theta_{i}} \ddot{a}_{\overline{x y}}=\sum_{k=0}^{\infty} v^{k} \frac{\partial}{\partial \theta_{1}}{ }_{k} p_{\overline{x y}} \\
& =\sum_{k=0}^{\infty} \frac{v^{k}}{[1-H(x, \infty)-H(\infty, y)+H(x, y)]^{2}}\left\{\begin{array}{l}
(H(x+k, y+k)-H(x+k, y)-H(x, y+k)+H(x, y)) \times \\
\left(\frac{\partial}{\partial \theta_{i}}\left(H(x, y)-F_{1}(x)-F_{2}(y)\right)\right)- \\
(1-H(x, \infty)-H(\infty, y)+H(x, y)) \times \\
\left(\frac{\partial}{\partial \theta_{i}}(H(x+k, y+k)-H(x, y+k)-H(x+k, y)+H(x, y))\right)
\end{array}\right\}
\end{aligned}
$$

Using equations (2.1) and (A.7), for $i=1,2$, we have:

$$
\begin{aligned}
\frac{\partial}{\partial m_{i}} F_{i}(x) & =-\exp \left[e^{-m_{i} / \sigma_{i}}\left(1-e^{x / \sigma_{i}}\right)\right] \cdot \frac{\partial}{\partial m_{i}}\left(e^{-m_{i} / \sigma_{i}}\left(1-e^{x / \sigma_{i}}\right)\right) \\
& =-\left(1-F_{i}(x)\right)\left(1-e^{x / \sigma_{i}}\right) \frac{\partial}{\partial m_{i}} e^{-m_{i} / \sigma_{i}} \\
& =\frac{1}{\sigma_{i}}\left(1-F_{i}(x)\right)\left(1-e^{x / \sigma_{i}}\right) e^{-m_{i} / \sigma_{i}} \\
& =f_{i}(0)-f_{i}(x)
\end{aligned}
$$

and $\frac{\partial}{\partial \sigma_{i}} F_{1}(x)=-\left(1-F_{1}(x)\right) \frac{\partial}{\partial \sigma_{i}}\left(e^{-m_{i} / \sigma_{1}}-e^{\left(x-m_{i}\right) / \sigma_{i}}\right)$

$$
\begin{aligned}
& =-\left(1-F_{i}(x)\right)\left(m_{i} e^{-m_{i} / \sigma_{i}}+\left(x-m_{i}\right) e^{\left(x-m_{i}\right) / \sigma_{i}}\right) / \sigma_{i}^{2} \\
& =\frac{-1}{\sigma_{i}}\left(m_{i} f_{i}(0)+\left(x-m_{i}\right) f_{i}(x)\right) \\
& =\frac{1}{\sigma_{i}}\left(\left(m_{i}-x\right) f_{i}(x)-m_{i} f_{i}(0)\right) .
\end{aligned}
$$

Equations (A.4), (A.5), (C.2) and (C.3) and the chain rule yield:

$$
\begin{aligned}
\frac{\partial}{\partial m_{1}} H(x, y) & =\frac{\partial}{\partial m_{1}} C\left(F_{1}(x), F_{2}(y)\right)=C_{1}\left(F_{1}(x), F_{2}(y)\right) \frac{\partial}{\partial m_{1}} F_{1}(x) \\
& =H_{1}(x, y)\left(f_{1}(0)-f_{1}(x)\right) / f_{1}(x) \\
\frac{\partial}{\partial m_{2}} H(x, y)= & H_{2}(x, y)\left(f_{2}(0)-f_{2}(y)\right) / f_{2}(y)
\end{aligned}
$$


and

$$
\begin{aligned}
\frac{\partial}{\partial \sigma_{1}} H(x, y) & =C_{1}\left(F_{1}(x), F_{2}(y)\right) \frac{\partial}{\partial \sigma_{1}} F_{1}(x) \\
& =H_{1}(x, y)\left(\left(m_{1}-x\right) f_{1}(x)-m_{1} f_{1}(0)\right) /\left(\sigma_{1} f_{1}(x)\right) \\
\frac{\partial}{\partial \sigma_{2}} H(x, y)= & H_{2}(x, y)\left(\left(m_{2}-y\right) f_{2}(y)-m_{2} f_{2}(0)\right) /\left(\sigma_{2} f_{2}(y)\right) .
\end{aligned}
$$

To calculate $(\partial / \partial \alpha) H(x, y)$, we use equation (3.2) and let $\mathrm{K}(\alpha)=\mathbf{K}=\exp (\alpha \mathrm{C}(u, \nu))=1+\frac{\left(e^{\alpha u}-1\right)\left(e^{\alpha v}-1\right)}{\left(e^{\alpha}-1\right)}$. Thus,

$$
\frac{\partial}{\partial \alpha} K(\alpha)=\frac{\left(e^{\alpha}-1\right)\left[u e^{(x u}\left(e^{\alpha u t}-1\right)+v e^{(\alpha)}\left(e^{\alpha u}-1\right)\right]-\left(e^{\alpha u}-1\right)\left(e^{\alpha v}-1\right) e^{\alpha}}{\left(e^{\alpha x}-1\right)^{2}}
$$

Making the substitutions:

$$
\begin{aligned}
e^{\alpha u}\left(e^{\alpha v}-1\right)= & C_{1}(u, v)\left(e^{\alpha x}-1\right) K \\
& \text { and } \\
e^{\alpha u v}\left(e^{\alpha u}-1\right)= & C_{2}(u, v)\left(e^{u x}-1\right) K,
\end{aligned}
$$

we have: $\frac{\partial}{\partial \alpha} K(\alpha)=u K C_{1}(u, v)+v K C_{2}(u, v)-\frac{e^{(\alpha}}{\left(e^{\alpha}-1\right)}(K-1)$

Define $C_{\alpha}(u, v)=\frac{\partial}{\partial \alpha} C(u, v)$. Thus, we have:

$$
\begin{aligned}
C_{\alpha}(u, v) & =\frac{\partial}{\partial \alpha}\left(\frac{\log (K)}{\alpha}\right)=\frac{\alpha \frac{1}{K} \frac{\partial K}{\partial \alpha}-\log (K)}{\alpha^{2}} \\
& =\frac{1}{\alpha} \frac{1}{K}\left(u K C_{1}(u, v)+v K C_{2}(u, v)+\frac{e^{\alpha x}}{\left(\mathrm{e}^{\prime \alpha}-1\right)}(1-K)\right)-\frac{1}{\alpha^{2}} \alpha C(u, v) .
\end{aligned}
$$

Rearranging terms, we then have:

$$
C_{\alpha}(u, v)=\frac{1}{\alpha}\left\{\frac{e^{\alpha}\left(e^{-\alpha(u, v)}-1\right)}{\left(e^{\alpha}-1\right)}+\left(u C_{1}(u, v)+v C_{2}(u, v)-C(u, v)\right)\right\} .
$$

Thus,

$$
\frac{\partial}{\partial \alpha} H(x, y)=\left.\frac{\partial}{\partial \alpha} C(u, v)\right|_{\left.u=F_{1}(x), v=F_{2}, y\right\}}=C_{\alpha}\left(F_{1}(x), F_{2}(y)\right) .
$$

To get the gradient of $\ddot{a}_{\bar{v} \bar{y}}(\theta)$, plug equations (C.4) to (C.8) into equation(C.1). 
APPENDIX D. Weibull Estimation Results

\begin{tabular}{ccccc}
\hline \multicolumn{5}{c}{ TABLE D.1. Weibull Parameter Estimates } \\
\hline \multirow{4}{*}{ Parameter } & Estimariate Distribution & \multicolumn{2}{c}{ Univariate Distributions } \\
\hline$m_{1}$ & 86.22 & Standard Error & Estimate & Standard Error \\
$\sigma_{1}$ & 10.16 & 0.27 & 86.73 & 0.28 \\
$m_{2}$ & 89.91 & 0.39 & 10.12 & 0.37 \\
$\sigma_{2}$ & 8.75 & 0.55 & 93.00 & 0.69 \\
$\alpha$ & -3.354 & 0.40 & 9.26 & 0.47 \\
& & 0.338 & Not Applicable & Not Applicable \\
\hline
\end{tabular}

TABLE D.2. Ratios of Dependent to Independent Joint and Last Annuity Values Based on Weibull Marginal Distributions.

Five Percent Interest is Assumed.

\begin{tabular}{c|ccccccc}
\hline \multirow{2}{*}{$\begin{array}{c}\text { Male } \\
\text { Age }\end{array}$} & \multicolumn{7}{|c}{ Female Age } \\
\cline { 2 - 8 } & 50 & 55 & 60 & 65 & 70 & 75 & 80 \\
\cline { 2 - 8 } 50 & 0.97 & 0.96 & 0.96 & 0.97 & 0.98 & 1.00 & 1.02 \\
55 & 0.97 & 0.96 & 0.95 & 0.95 & 0.97 & 0.99 & 1.02 \\
60 & 0.97 & 0.96 & 0.95 & 0.94 & 0.95 & 0.97 & 1.01 \\
65 & 0.98 & 0.97 & 0.95 & 0.94 & 0.94 & 0.95 & 0.99 \\
70 & 0.99 & 0.98 & 0.96 & 0.95 & 0.93 & 0.93 & 0.96 \\
75 & 1.00 & 0.99 & 0.99 & 0.97 & 0.95 & 0.94 & 0.94 \\
80 & 1.01 & 1.01 & 1.01 & 1.00 & 0.99 & 0.96 & 0.94 \\
\hline
\end{tabular}




\section{APPENDLX E \\ Spearman's Test of Independence}

In this appendix, we show how to apply Spearman's test of independence on our data, assuming that the marginal distributions are known. This last assumption is reasonable because extensive information is available on the law of mortality for individual lives. The following technique is a quick nonparametric way of identifying and measuring the dependence. The results from this method must be used with caution because we are assuming that the marginals are known.

Suppose we observe the time of deaths $\left(T_{1, k}, T_{2, k}\right)$ for $k=1,2, \ldots, n$ where $T_{i, k}$ has a known continuous distribution function $G_{i, k}(t), t>0, i=1,2$. In our case, these distributions will not be identical. Consider the uniform random variables $U_{i, k} \equiv G_{i, k}\left(T_{i, k}\right)$. Assume that the pairs $\left(U_{1, k}, U_{2, k}\right)$ for $k=1,2, \ldots, n$ are independent and identically distributed with a common copula $C(u, v)$. The assumption of a common copula allows us to calculate Spearman's sample correlation coefficient and use it to test independence, that is $C(u, v)=u v$. Let $R_{i, k}$ denote the rank of $U_{i, k}$, then Spearman's correlation is

$$
\widehat{\rho}=\frac{\sum_{k=1}^{n}\left[R_{1, k}-(n+1) / 2\right]\left[R_{2, k}-(n+1) / 2\right]}{n\left(n^{2}-1\right) / 12}
$$

An estimate of the asymptotic variance of this statistic is $(n-1)^{-1}$. So we would reject the null hypothesis of independence at a $5 \%$ level, if $|\widehat{\rho}|>1.96(n-1)^{-1 / 2}$.

Let's apply this technique to our data where $n=229$ policies had both annuitants die during the observation period. Let $F_{i}(x)$ denote a Gompertz distribution as defined in (2.1) with parameters $m_{i}, \sigma_{i}$. If $i=1$ then these parameters refer to a male life and if $i=2$ then they refer to a female life. Consulting Table 2, we let $m_{1}=86.38, \sigma_{1}=9.83$ and $m_{2}=92.17, \sigma_{2}=8.11$. We found that the estimate of Spearman's correlation coefficient did not change very much when other reasonable parameter values were used. In our case, $G_{i, k}$ is the distribution of the time of death, given that the death occurs during the observation period. Let $x_{i, k}+a_{k}$ denote the age at the start of the observation period and let $x_{i, k}+a_{k}+b_{k}$ denote the age at the end of the observation period, then

$$
G_{i, k}(t)=\frac{F_{i}\left(x_{i, k}+t\right)-F_{i}\left(x_{i, k}+a_{k}\right)}{F_{i}\left(x_{i, k}+a_{k}+b_{k}\right)-F_{i}\left(x_{i, k}+a_{k}\right)}
$$

Using our data, we found that the sample correlation is $\hat{\rho}=.414$ and a $95 \%$ confidence interval is $(.282,547)$. Note that our estimate of $\rho$, given in section 3.2 , is equal to 49 and that this lies within this confidence interval. 


\section{APPENDIX F \\ Miscellaneous Tables and Graphs}

Tables F.1 to F.5 describe the joint annuity data by providing a breakdown of the total number of policies by status, sex, and issue age groups. For status 1, both the primary and the secondary annuitants are alive as of the end of the observation period. For status 2 , only the primary annuitant is alive. For status 3 , only the secondary annuitant is alive. For status 4 , both the annuitants are alive.

Table F.6 provides some meaningful statistics on issue ages.

Tables F.7 to F.16 provide joint and last-survivor annuity values. The assumption of independence and the corresponding ratios of dependent to independent assumptions calculated using the guarantee likelihood result for various discount interest rates are presented.

Figures F.1 and F.2 provide graphs of the Kaplan-Meier fitted female distributions versus the Gompertz and the Weibull distributions. Figures F.3 and F.4 provide graphs of the KaplanMeier and the logistic distribution for male and female. 
Table F.1 Number of Policies by Sex and Age Group

Status 1 - Both Primary and Secondary Annuitants are Alive

\begin{tabular}{|c|c|c|c|c|c|c|c|c|}
\hline \multirow{3}{*}{$\begin{array}{l}\text { Age } \\
\text { Group }\end{array}$} & \multicolumn{4}{|c|}{ Primary Annuitants } & \multicolumn{4}{|c|}{ Secondary Annuitants } \\
\hline & \multicolumn{2}{|c|}{ Male } & \multicolumn{2}{|c|}{ Female } & \multicolumn{2}{|c|}{ Male } & \multicolumn{2}{|c|}{ Female } \\
\hline & Count & $\%$ of Total & Count & $\%$ of Total & Count & $\%$ of Total & Count & $\%$ of Total \\
\hline$<60$ & 1,524 & $14.6 \%$ & 330 & $12.6 \%$ & 241 & $9.3 \%$ & 3,676 & $35.2 \%$ \\
\hline $60-70$ & 7,120 & $68.3 \%$ & 1,807 & $69.1 \%$ & 1,659 & $63.8 \%$ & 5,916 & $56.7 \%$ \\
\hline $70-80$ & 1,729 & $16.6 \%$ & 474 & $18.1 \%$ & 679 & $26.1 \%$ & 808 & $7.7 \%$ \\
\hline$>=80$ & 54 & $0.5 \%$ & 4 & $0.2 \%$ & 22 & $0.8 \%$ & 41 & $0.4 \%$ \\
\hline Total & 10,427 & & 2,615 & & 2,601 & & 10,441 & \\
\hline
\end{tabular}

Table F.2 Number of Policies by Sex and Age Group

Status 2 - Only the Primary Annuitant is Alive

\begin{tabular}{|c|c|c|c|c|c|c|c|c|}
\hline \multirow{3}{*}{$\begin{array}{r}\text { Age } \\
\text { Group }\end{array}$} & \multicolumn{4}{|c|}{ Primary Annuitants } & \multicolumn{4}{|c|}{ Secondary Annuitants } \\
\hline & \multicolumn{2}{|c|}{ Male } & \multicolumn{2}{|c|}{ Female } & \multicolumn{2}{|c|}{ Male } & \multicolumn{2}{|c|}{ Female } \\
\hline & Count & $\%$ of Total & Count & $\%$ of Total & Count & $\%$ of Total & Count & $\%$ of Total \\
\hline$<60$ & 14 & $6.0 \%$ & 9 & $6.1 \%$ & 8 & $5.4 \%$ & 31 & $13.4 \%$ \\
\hline $60 \cdot 70$ & 146 & $62.9 \%$ & 101 & $68.7 \%$ & 79 & $53.4 \%$ & 150 & $64.9 \%$ \\
\hline $70-80$ & 70 & $30.2 \%$ & 37 & $25.2 \%$ & 60 & $40.5 \%$ & 50 & $21.6 \%$ \\
\hline$>=80$ & 2 & $0.9 \%$ & 0 & $0.0 \%$ & 1 & $0.7 \%$ & 0 & $0.0 \%$ \\
\hline Total & 232 & & 147 & & 148 & & 231 & \\
\hline
\end{tabular}

Table F.3 Number of Policies by Sex and Age Group

Status 3 - Only the Secondary Annuitant is Alive

\begin{tabular}{|c|c|c|c|c|c|c|c|c|}
\hline \multirow{3}{*}{$\begin{array}{r}\text { Age } \\
\text { Group }\end{array}$} & \multicolumn{4}{|c|}{ Primary Annuitants } & \multicolumn{4}{|c|}{ Secondary Annuitants } \\
\hline & \multicolumn{2}{|c|}{ Male } & \multicolumn{2}{|c|}{ Female } & \multicolumn{2}{|c|}{ Male } & \multicolumn{2}{|c|}{ Female } \\
\hline & Count & of Total & Count & $\%$ of Total & Count & $\%$ of Total & Count & $\%$ of Total \\
\hline$<60$ & 68 & $5.8 \%$ & 7 & $6.1 \%$ & 8 & $7.0 \%$ & 286 & $24.2 \%$ \\
\hline $60-70$ & 722 & $61.2 \%$ & 78 & $67.8 \%$ & 60 & $52.6 \%$ & 731 & $61.9 \%$ \\
\hline $70-80$ & 368 & $31.2 \%$ & 30 & $26.1 \%$ & 46 & $40.4 \%$ & 154 & $13.0 \%$ \\
\hline$>=80$ & 22 & $1.9 \%$ & 0 & $0.0 \%$ & 0 & $0.0 \%$ & 10 & $0.8 \%$ \\
\hline Total & 1,180 & & 115 & & 114 & & 1,181 & \\
\hline
\end{tabular}


Table F.4 Number of Policies by Sex and Age Group Status 4- Both Primary and Secondary Annuitants are Dead

\begin{tabular}{|c|c|c|c|c|c|c|c|c|}
\hline \multirow{3}{*}{$\begin{array}{l}\text { Age } \\
\text { Group }\end{array}$} & \multicolumn{4}{|c|}{ Primary Annuitants } & \multicolumn{4}{|c|}{ Secondary Annuitants } \\
\hline & \multicolumn{2}{|c|}{ Male } & \multicolumn{2}{|c|}{ Female } & \multicolumn{2}{|c|}{ Male } & \multicolumn{2}{|c|}{ Female } \\
\hline & Count & $\%$ of Total & Count & $\%$ of Total & Count & \% of Total & Count & $\%$ of Total \\
\hline$<60$ & 6 & $3.0 \%$ & 2 & $5.9 \%$ & 1 & $2.9 \%$ & 19 & $9.6 \%$ \\
\hline $60-70$ & 106 & $53.8 \%$ & 20 & $58.8 \%$ & 14 & $41.2 \%$ & 130 & $66.0 \%$ \\
\hline $70-80$ & 79 & $40.1 \%$ & 11 & $32.4 \%$ & 17 & $50.0 \%$ & 42 & $21.3 \%$ \\
\hline$>=80$ & 6 & $3.0 \%$ & 1 & $2.9 \%$ & 2 & $5.9 \%$ & 6 & $3.0 \%$ \\
\hline Total & 197 & & 34 & & 34 & & 197 & \\
\hline
\end{tabular}

Table F.5 Number of Policies by Sex and Age Group

All Status

\begin{tabular}{|c|c|c|c|c|c|c|c|c|}
\hline \multirow{3}{*}{$\begin{array}{l}\text { Age } \\
\text { Group }\end{array}$} & \multicolumn{4}{|c|}{ Primary Annuitants } & \multicolumn{4}{|c|}{ Secondary Annuitants } \\
\hline & \multicolumn{2}{|c|}{ Male } & \multicolumn{2}{|c|}{ Female } & \multicolumn{2}{|c|}{ Male } & \multicolumn{2}{|c|}{ Female } \\
\hline & Count & $\%$ of Total & Count & $\%$ of Total & Count & $\%$ of Total & Count & $\%$ of Total \\
\hline$<60$ & 1,612 & $13.4 \%$ & 348 & $12.0 \%$ & 258 & $8.9 \%$ & 4,012 & $33.3 \%$ \\
\hline $60-70$ & 8,094 & $67.2 \%$ & 2,006 & $68.9 \%$ & 1,812 & $62.5 \%$ & 6,927 & $57.5 \%$ \\
\hline $70-80$ & 2,246 & $18.7 \%$ & 552 & $19.0 \%$ & 802 & $27.7 \%$ & 1,054 & $8.7 \%$ \\
\hline$>=80$ & 84 & $0.7 \%$ & 5 & $0.2 \%$ & 25 & $0.9 \%$ & 57 & $0.5 \%$ \\
\hline Total & 12,036 & & 2,911 & & 2,897 & & 12,050 & \\
\hline
\end{tabular}

Table F.6 Some Basic Statistics on Issue Ages

\begin{tabular}{lr|r|r} 
& \multicolumn{3}{c}{ Primary Annuitants } \\
\cline { 2 - 4 } Male & $\begin{array}{r}\text { Average } \\
\text { Issue Age }\end{array}$ & $\begin{array}{r}\text { Minimum } \\
\text { Issue Age }\end{array}$ & $\begin{array}{r}\text { Maximum } \\
\text { Issue Age }\end{array}$ \\
\cline { 2 - 4 } Female & 65.2 & 0.1 & 90.7 \\
65.2 & 31.4 & 88.9
\end{tabular}

Male

Female

\begin{tabular}{r|r|r}
\multicolumn{3}{|c}{ Secondary Annuitants } \\
\hline $\begin{array}{r}\text { Average } \\
\text { Issue Age }\end{array}$ & $\begin{array}{r}\text { Minimum } \\
\text { Issue Age }\end{array}$ & $\begin{array}{r}\text { Maximum } \\
\text { Issue Age }\end{array}$ \\
\hline 66.8 & 9.3 & 91.5 \\
62.0 & 0.3 & 89.7
\end{tabular}


Table F.7 Joint and Last Survivor Annuity Values Using Independent Assumptions Based on Guarantee Period Likelihood Estimates 0\% Interest Rate

\begin{tabular}{|c|c|c|c|c|c|c|c|c|c|c|}
\hline Male & \multicolumn{9}{|c|}{ Female Age } & \multirow[b]{2}{*}{95} \\
\hline Age & 50 & 55 & 60 & 65 & 70 & 75 & 80 & 85 & 90 & \\
\hline 50 & 41.51 & 38.56 & 36.31 & 34.71 & 33.63 & 32.77 & 31.65 & 29.86 & 27.24 & 23.85 \\
\hline 55 & 40.18 & 36.61 & 33.70 & 31.50 & 29.96 & 28.93 & 28.11 & 27.03 & 25.25 & 22.60 \\
\hline 60 & 39.33 & 35.28 & 31.77 & 28.93 & 26.80 & 25.34 & 24.40 & 23.64 & 22.59 & 20.81 \\
\hline 65 & 38.82 & 34.45 & 30.47 & 27.04 & 24.30 & 22.29 & 20.95 & 20.11 & 19.43 & 18.41 \\
\hline 70 & 38.41 & 33.96 & 29.66 & 25.77 & 22.47 & 19.88 & 18.04 & 16.86 & 16.15 & 15.55 \\
\hline 75 & 37.59 & 33.55 & 29.18 & 24.99 & 21.26 & 18.15 & 15.78 & 14.16 & 13.18 & 12.61 \\
\hline 80 & 35.85 & 32.74 & 28.79 & 24.54 & 20.52 & 17.02 & 14.18 & 12.11 & 10.76 & 9.98 \\
\hline 85 & 33.05 & 30.99 & 27.98 & 24.16 & 20.11 & 16.35 & 13.16 & 10.68 & 8.95 & 7.90 \\
\hline 90 & 29.41 & 28.19 & 26.22 & 23.36 & 19.76 & 15.98 & 12.57 & 9.79 & 7.74 & 6.40 \\
\hline 95 & 25.18 & 24.53 & 23.40 & 21.59 & 18.94 & 15.65 & 12.25 & 9.30 & 7.01 & 5.41 \\
\hline
\end{tabular}

Table F.8 Ratios of Dependent to Independent Joint and Last Survivor Annuity Values Based on Guarantee Period Likelihood Estimates 0\% Interest Rate

\begin{tabular}{c|cccccccccc}
\hline Male & \multicolumn{10}{|c}{ Female Age } \\
\cline { 2 - 11 } Age & $\mathbf{5 0}$ & $\mathbf{5 5}$ & $\mathbf{6 0}$ & $\mathbf{6 5}$ & $\mathbf{7 0}$ & $\mathbf{7 5}$ & $\mathbf{8 0}$ & $\mathbf{8 5}$ & $\mathbf{9 0}$ & $\mathbf{9 5}$ \\
\cline { 2 - 11 } $\mathbf{5 0}$ & 0.92 & 0.91 & 0.92 & 0.93 & 0.95 & 0.98 & 1.01 & 1.05 & 1.07 & 1.06 \\
$\mathbf{5 5}$ & 0.92 & 0.91 & 0.90 & 0.91 & 0.93 & 0.96 & 1.00 & 1.05 & 1.09 & 1.09 \\
$\mathbf{6 0}$ & 0.94 & 0.92 & 0.90 & 0.90 & 0.91 & 0.93 & 0.98 & 1.04 & 1.11 & 1.13 \\
$\mathbf{6 5}$ & 0.95 & 0.94 & 0.92 & 0.90 & 0.89 & 0.91 & 0.95 & 1.02 & 1.10 & 1.16 \\
$\mathbf{7 0}$ & 0.97 & 0.96 & 0.94 & 0.92 & 0.90 & 0.89 & 0.92 & 0.98 & 1.07 & 1.16 \\
$\mathbf{7 5}$ & 1.00 & 0.98 & 0.97 & 0.95 & 0.93 & 0.90 & 0.89 & 0.93 & 1.02 & 1.12 \\
$\mathbf{8 0}$ & 1.03 & 1.02 & $\mathbf{1 . 0 1}$ & 1.00 & 0.98 & 0.94 & 0.90 & 0.90 & 0.95 & 1.04 \\
$\mathbf{8 5}$ & 1.04 & 1.06 & $\mathbf{1 . 0 6}$ & 1.05 & 1.03 & 1.00 & 0.95 & 0.90 & 0.89 & 0.95 \\
$\mathbf{9 0}$ & 1.04 & $\mathbf{1 . 0 6}$ & $\mathbf{1 . 0 8}$ & 1.10 & $\mathbf{1 . 0 9}$ & 1.07 & $\mathbf{1 . 0 2}$ & 0.95 & 0.88 & 0.87 \\
$\mathbf{9 5}$ & 1.02 & $\mathbf{1 . 0 4}$ & 1.07 & 1.11 & $\mathbf{1 . 1 3}$ & 1.12 & 1.08 & 1.00 & 0.90 & 0.84 \\
\hline
\end{tabular}


Table F.9 Joint and Last Survivor Annuity Values Using Independent Assumptions Based on Guarantee Period Likelihood Estimates $5 \%$ Interest Rate

\begin{tabular}{|c|c|c|c|c|c|c|c|c|c|c|}
\hline Male & & & & & nale $A$ & & & & & \\
\hline Age & 50 & 55 & 60 & 65 & 70 & 75 & 80 & 85 & 90 & 95 \\
\hline 50 & 18.03 & 17.58 & 17.17 & 16.81 & 16.52 & 16.28 & 16.03 & 15.68 & 15.11 & 14.22 \\
\hline 55 & 17.81 & 17.24 & 16.68 & 16.17 & 15.74 & 15.40 & 15.12 & 14.84 & 14.42 & 13.70 \\
\hline 60 & 17.64 & 16.96 & 16.26 & 15.57 & 14.96 & 14.46 & 14.07 & 13.78 & 13.46 & 12.94 \\
\hline 65 & 17.51 & 16.75 & 15.92 & 15.06 & 14.24 & 13.52 & 12.96 & 12.56 & 12.25 & 11.90 \\
\hline 70 & 17.42 & 16.60 & 15.66 & 14.65 & 13.62 & 12.68 & 11.88 & 11.29 & 10.89 & 10.59 \\
\hline 75 & 17.30 & 16.49 & 15.48 & 14.35 & 13.15 & 11.97 & 10.92 & 10.09 & 9.51 & 9.15 \\
\hline 80 & 17.05 & 16.34 & 15.35 & 14.15 & 12.81 & 11.44 & 10.14 & 9.05 & 8.24 & 7.72 \\
\hline 85 & 16.60 & 16.03 & 15.17 & 14.00 & 12.59 & 11.07 & 9.58 & 8.24 & 7.18 & 6.46 \\
\hline 90 & 15.85 & 15.45 & 14.79 & 13.79 & 12.43 & 10.85 & 9.21 & 7.67 & 6.39 & 5.46 \\
\hline 95 & 14.77 & 14.51 & 14.05 & 13.30 & 12.18 & 10.68 & 8.99 & 7.33 & 5.87 & 4.74 \\
\hline
\end{tabular}

Table F.10 Ratios of Dependent to Independent Joint and Last Survivor Annuity Values Based on Guarantee Period Likelihood Estimates 5\% Interest Rate

\begin{tabular}{c|cccccccccc}
\hline Male & \multicolumn{10}{|c}{ Female Age } \\
\cline { 2 - 11 } Age & $\mathbf{5 0}$ & $\mathbf{5 5}$ & $\mathbf{6 0}$ & $\mathbf{6 5}$ & $\mathbf{7 0}$ & $\mathbf{7 5}$ & $\mathbf{8 0}$ & $\mathbf{8 5}$ & $\mathbf{9 0}$ & $\mathbf{9 5}$ \\
\cline { 2 - 11 } $\mathbf{5 0}$ & 0.97 & 0.96 & 0.96 & 0.96 & 0.97 & 0.99 & 1.01 & $\mathbf{1 . 0 3}$ & 1.04 & $\mathbf{1 . 0 4}$ \\
$\mathbf{5 5}$ & 0.97 & 0.96 & 0.95 & 0.95 & 0.96 & 0.97 & 1.00 & 1.03 & 1.06 & $\mathbf{1 . 0 6}$ \\
$\mathbf{6 0}$ & 0.97 & 0.96 & 0.95 & 0.94 & 0.94 & 0.96 & 0.99 & 1.03 & 1.07 & $\mathbf{1 . 0 9}$ \\
$\mathbf{6 5}$ & 0.98 & 0.97 & 0.96 & 0.94 & 0.93 & 0.94 & 0.97 & 1.01 & 1.07 & $\mathbf{1 . 1 1}$ \\
$\mathbf{7 0}$ & $\mathbf{0 . 9 9}$ & 0.98 & 0.97 & 0.95 & 0.94 & 0.93 & 0.94 & 0.99 & 1.05 & $\mathbf{1 . 1 2}$ \\
$\mathbf{7 5}$ & $\mathbf{1 . 0 0}$ & $\mathbf{1 . 0 0}$ & $\mathbf{0 . 9 9}$ & 0.98 & 0.96 & 0.93 & 0.92 & 0.95 & 1.02 & $\mathbf{1 . 0 9}$ \\
$\mathbf{8 0}$ & $\mathbf{1 . 0 2}$ & $\mathbf{1 . 0 2}$ & 1.01 & 1.01 & 0.99 & 0.96 & 0.93 & 0.92 & 0.96 & $\mathbf{1 . 0 4}$ \\
$\mathbf{8 5}$ & 1.02 & 1.03 & 1.04 & 1.04 & 1.03 & 1.01 & 0.97 & 0.93 & 0.92 & 0.96 \\
$\mathbf{9 0}$ & 1.02 & 1.04 & 1.05 & 1.07 & 1.07 & 1.06 & 1.03 & 0.97 & 0.90 & 0.89 \\
$\mathbf{9 5}$ & 1.02 & $\mathbf{1 . 0 3}$ & 1.05 & 1.07 & $\mathbf{1 . 1 0}$ & 1.10 & 1.07 & 1.01 & 0.92 & 0.86 \\
\hline
\end{tabular}


Table F.11 Joint and Last Survivor Annuity Values Using Independent Assumptions Based on Guarantee Period Likelihood Estimates $10 \%$ Interest Rate

\begin{tabular}{c|cccccccccc}
\hline Male & \multicolumn{10}{|c}{ Female Age } \\
\cline { 2 - 11 } Age & $\mathbf{5 0}$ & $\mathbf{5 5}$ & $\mathbf{6 0}$ & $\mathbf{6 5}$ & $\mathbf{7 0}$ & $\mathbf{7 5}$ & $\mathbf{8 0}$ & $\mathbf{8 5}$ & $\mathbf{9 0}$ & $\mathbf{9 5}$ \\
\cline { 2 - 11 } $\mathbf{5 0}$ & 10.72 & $\mathbf{1 0 . 6 3}$ & 10.54 & $10 . \mathbf{4 4}$ & 10.35 & 10.26 & 10.18 & 10.08 & 9.94 & $\mathbf{9 . 6 8}$ \\
$\mathbf{5 5}$ & 10.67 & $\mathbf{1 0 . 5 6}$ & 10.43 & 10.29 & 10.15 & 10.01 & 9.90 & 9.79 & 9.66 & $9 . \mathbf{4 4}$ \\
$\mathbf{6 0}$ & 10.63 & $\mathbf{1 0 . 4 9}$ & 10.32 & 10.12 & 9.91 & 9.71 & 9.54 & 9.39 & 9.26 & 9.08 \\
$\mathbf{6 5}$ & 10.59 & 10.43 & 10.21 & $\mathbf{9 . 9 5}$ & 9.67 & 9.38 & 9.12 & 8.90 & 8.73 & 8.57 \\
$\mathbf{7 0}$ & 10.56 & 10.37 & 10.12 & 9.80 & 9.43 & 9.03 & 8.66 & 8.33 & 8.09 & 7.91 \\
$\mathbf{7 5}$ & 10.53 & 10.33 & 10.04 & 9.67 & 9.22 & 8.71 & 8.20 & 7.74 & 7.37 & $\mathbf{7 . 1 2}$ \\
$\mathbf{8 0}$ & $\mathbf{1 0 . 4 9}$ & 10.28 & 9.98 & $\mathbf{9 . 5 7}$ & 9.05 & 8.43 & 7.78 & 7.16 & 6.65 & $\mathbf{6 . 2 8}$ \\
$\mathbf{8 5}$ & $\mathbf{1 0 . 4 0}$ & 10.22 & 9.92 & $\mathbf{9 . 4 9}$ & 8.92 & 8.22 & 7.45 & 6.67 & 5.98 & $\mathbf{5 . 4 7}$ \\
$\mathbf{9 0}$ & $\mathbf{1 0 . 2 3}$ & 10.08 & 9.82 & $\mathbf{9 . 4 2}$ & 8.83 & 8.08 & 7.20 & 6.29 & 5.44 & $\mathbf{4 . 7 7}$ \\
$\mathbf{9 5}$ & 9.93 & 9.82 & 9.61 & 9.27 & 8.73 & $\mathbf{7 . 9 8}$ & $\mathbf{7 . 0 5}$ & 6.04 & 5.05 & $\mathbf{4 . 2 3}$ \\
\hline
\end{tabular}

Table F.12 Ratios of Dependent to Independent Joint and Last Survivor Annuity Values Based on Guarantee Period Likelihood Estimates $10 \%$ Interest Rate

\begin{tabular}{c|ccccccccccc}
\hline Male & \multicolumn{10}{|c}{ Female Age } \\
\cline { 2 - 11 } Age & $\mathbf{5 0}$ & $\mathbf{5 5}$ & $\mathbf{6 0}$ & $\mathbf{6 5}$ & $\mathbf{7 0}$ & $\mathbf{7 5}$ & $\mathbf{8 0}$ & $\mathbf{8 5}$ & $\mathbf{9 0}$ & $\mathbf{9 5}$ \\
\cline { 2 - 11 } & 0.99 & 0.98 & 0.98 & 0.98 & 0.98 & 0.99 & 1.00 & 1.02 & 1.03 & 1.03 \\
$\mathbf{5 5}$ & 0.99 & 0.98 & 0.98 & 0.97 & 0.98 & 0.98 & 1.00 & 1.02 & 1.04 & 1.04 \\
$\mathbf{6 0}$ & 0.99 & 0.98 & 0.98 & 0.97 & 0.97 & 0.97 & 0.99 & 1.02 & 1.05 & 1.06 \\
$\mathbf{6 5}$ & 0.99 & 0.99 & 0.98 & 0.97 & 0.96 & 0.96 & 0.98 & 1.01 & 1.05 & 1.08 \\
$\mathbf{7 0}$ & 1.00 & 0.99 & 0.98 & 0.97 & 0.96 & 0.95 & 0.96 & 0.99 & 1.04 & 1.09 \\
$\mathbf{7 5}$ & 1.00 & 1.00 & $\mathbf{1 . 0 0}$ & 0.99 & 0.97 & 0.95 & 0.94 & 0.96 & 1.01 & 1.07 \\
$\mathbf{8 0}$ & 1.01 & 1.01 & $\mathbf{1 . 0 1}$ & 1.01 & 1.00 & 0.98 & 0.95 & 0.94 & 0.97 & 1.03 \\
$\mathbf{8 5}$ & $\mathbf{1 . 0 1}$ & 1.02 & $\mathbf{1 . 0 2}$ & 1.03 & 1.03 & $\mathbf{1 . 0 1}$ & 0.98 & 0.94 & 0.93 & 0.97 \\
$\mathbf{9 0}$ & 1.01 & 1.02 & 1.03 & 1.04 & 1.05 & $\mathbf{1 . 0 5}$ & 1.03 & 0.98 & 0.92 & 0.91 \\
$\mathbf{9 5}$ & $\mathbf{1 . 0 1}$ & 1.02 & $\mathbf{1 . 0 3}$ & 1.05 & 1.07 & $\mathbf{1 . 0 8}$ & 1.06 & 1.01 & 0.94 & 0.88 \\
\hline
\end{tabular}


Table F.13 Joint and Last Survivor Annuity Values Using Independent Assumptions Based on Guarantee Period Likelihood Estimates

$15 \%$ Interest Rate

\begin{tabular}{|c|c|c|c|c|c|c|c|c|c|c|}
\hline Male & & & & & nale & & & & & \\
\hline Age & 50 & 55 & 60 & 65 & 70 & 75 & 80 & 85 & 90 & 95 \\
\hline 50 & 7.62 & 7.60 & 7.58 & 7.54 & 7.51 & 7.47 & 7.44 & 7.40 & 7.35 & 7.26 \\
\hline 55 & 7.61 & 7.58 & 7.54 & 7.50 & 7.44 & 7.39 & 7.33 & 7.28 & 7.22 & 7.14 \\
\hline 60 & 7.60 & 7.56 & 7.51 & 7.44 & 7.36 & 7.27 & 7.18 & 7.10 & 7.03 & 6.95 \\
\hline 65 & 7.58 & 7.54 & 7.47 & 7.38 & 7.26 & 7.13 & 7.00 & 6.87 & 6.77 & 6.68 \\
\hline 70 & 7.57 & 7.51 & 7.43 & 7.31 & 7.16 & 6.97 & 6.78 & 6.59 & 6.43 & 6.31 \\
\hline 75 & 7.56 & 7.49 & 7.39 & 7.25 & 7.05 & 6.81 & 6.53 & 6.26 & 6.02 & 5.84 \\
\hline 80 & 7.55 & 7.48 & 7.36 & 7.20 & 6.96 & 6.66 & 6.30 & 5.92 & 5.58 & 5.31 \\
\hline 85 & 7.52 & 7.45 & 7.34 & 7.15 & 6.88 & 6.52 & 6.08 & 5.60 & 5.14 & 4.76 \\
\hline 90 & 7.48 & 7.42 & 7.30 & 7.11 & 6.83 & 6.43 & 5.92 & 5.34 & 4.75 & 4.25 \\
\hline 95 & 7.39 & 7.33 & 7.23 & 7.06 & 6.77 & 6.35 & 5.80 & 5.14 & 4.45 & 3.83 \\
\hline
\end{tabular}

Table F.14 Ratios of Dependent to Independent Joint and Last Survivor Annuity Values Based on Guarantee Period Likelihood Estimates 15\% Interest Rate

\begin{tabular}{c|cccccccccc}
\hline Male & \multicolumn{10}{|c}{ Female Age } \\
\cline { 2 - 11 } Age & $\mathbf{5 0}$ & $\mathbf{5 5}$ & $\mathbf{6 0}$ & $\mathbf{6 5}$ & $\mathbf{7 0}$ & $\mathbf{7 5}$ & $\mathbf{8 0}$ & $\mathbf{8 5}$ & $\mathbf{9 0}$ & $\mathbf{9 5}$ \\
\cline { 2 - 11 } $\mathbf{5 0}$ & 1.00 & 0.99 & 0.99 & 0.99 & 0.99 & 0.99 & 1.00 & 1.01 & 1.02 & 1.02 \\
$\mathbf{5 5}$ & 0.99 & 0.99 & 0.99 & 0.99 & 0.99 & 0.99 & 1.00 & 1.01 & 1.02 & 1.03 \\
$\mathbf{6 0}$ & 1.00 & 0.99 & 0.99 & 0.98 & 0.98 & 0.98 & 0.99 & 1.01 & 1.03 & 1.04 \\
$\mathbf{6 5}$ & 1.00 & 0.99 & 0.99 & 0.98 & 0.97 & 0.97 & 0.98 & 1.00 & 1.03 & 1.06 \\
$\mathbf{7 0}$ & 1.00 & 1.00 & 0.99 & 0.98 & 0.97 & 0.97 & 0.97 & 0.99 & 1.03 & 1.07 \\
$\mathbf{7 5}$ & 1.00 & 1.00 & 1.00 & 0.99 & 0.98 & 0.97 & 0.96 & 0.97 & 1.01 & 1.06 \\
$\mathbf{8 0}$ & 1.00 & 1.01 & 1.01 & 1.00 & 1.00 & 0.98 & 0.96 & 0.95 & 0.98 & 1.03 \\
$\mathbf{8 5}$ & 1.01 & 1.01 & 1.01 & 1.02 & 1.02 & 1.01 & 0.99 & 0.96 & 0.94 & 0.97 \\
$\mathbf{9 0}$ & 1.01 & 1.01 & 1.02 & 1.03 & 1.04 & 1.04 & 1.02 & 0.98 & 0.94 & 0.92 \\
$\mathbf{9 5}$ & 1.01 & 1.01 & 1.02 & 1.04 & 1.05 & 1.06 & 1.05 & 1.02 & 0.95 & 0.89 \\
\hline
\end{tabular}


Table F.15 Joint and Last Survivor Annuity Values Using Independent Assumptions Based on Guarantee Period Likelihood Estimates $20 \%$ Interest Rate

\begin{tabular}{c|cccccccccc}
\hline Male & \multicolumn{10}{|c}{ Female Age } \\
\cline { 2 - 11 } Age & $\mathbf{5 0}$ & $\mathbf{5 5}$ & $\mathbf{6 0}$ & $\mathbf{6 5}$ & $\mathbf{7 0}$ & $\mathbf{7 5}$ & $\mathbf{8 0}$ & $\mathbf{8 5}$ & $\mathbf{9 0}$ & $\mathbf{9 5}$ \\
\cline { 2 - 11 } $\mathbf{5 0}$ & 5.99 & 5.98 & 5.97 & 5.96 & 5.95 & 5.93 & 5.91 & 5.89 & 5.87 & 5.84 \\
$\mathbf{5 5}$ & 5.99 & 5.98 & 5.96 & $\mathbf{5 . 9 5}$ & 5.92 & 5.89 & 5.86 & 5.83 & $\mathbf{5 . 8 0}$ & 5.77 \\
$\mathbf{6 0}$ & 5.98 & 5.97 & 5.95 & 5.92 & 5.89 & 5.84 & 5.80 & 5.75 & 5.70 & 5.66 \\
$\mathbf{6 5}$ & 5.98 & 5.96 & 5.93 & 5.90 & 5.84 & 5.78 & 5.70 & 5.63 & 5.56 & 5.50 \\
$\mathbf{7 0}$ & 5.97 & 5.95 & 5.92 & $\mathbf{5 . 8 6}$ & 5.79 & 5.70 & 5.59 & 5.47 & $\mathbf{5 . 3 6}$ & $\mathbf{5 . 2 7}$ \\
$\mathbf{7 5}$ & 5.96 & 5.94 & 5.90 & 5.83 & $\mathbf{5 . 7 4}$ & 5.61 & 5.45 & 5.27 & 5.11 & $\mathbf{4 . 9 8}$ \\
$\mathbf{8 0}$ & 5.96 & 5.93 & 5.88 & 5.80 & 5.68 & 5.51 & 5.30 & 5.06 & 4.82 & $\mathbf{4 . 6 2}$ \\
$\mathbf{8 5}$ & 5.95 & 5.92 & 5.86 & 5.77 & 5.63 & 5.43 & 5.16 & 4.84 & 4.51 & $\mathbf{4 . 2 3}$ \\
$\mathbf{9 0}$ & 5.94 & $\mathbf{5 . 9 0}$ & 5.85 & $\mathbf{5 . 7 5}$ & 5.59 & 5.36 & $\mathbf{5 . 0 4}$ & $\mathbf{4 . 6 5}$ & $\mathbf{4 . 2 3}$ & $\mathbf{3 . 8 4}$ \\
$\mathbf{9 5}$ & 5.91 & 5.88 & 5.82 & $\mathbf{5 . 7 2}$ & 5.56 & 5.31 & $\mathbf{4 . 9 5}$ & $\mathbf{4 . 5 0}$ & $\mathbf{4 . 0 0}$ & $\mathbf{3 . 5 1}$ \\
\hline
\end{tabular}

Table F.16 Ratios of Dependent to Independent Joint and Last Survivor Annuity Values Based on Guarantee Period Likelihood Estimates 20\% Interest Rate

\begin{tabular}{c|cccccccccc}
\hline Male & \multicolumn{10}{|c}{ Female Age } \\
\cline { 2 - 11 } Age & $\mathbf{5 0}$ & $\mathbf{5 5}$ & $\mathbf{6 0}$ & $\mathbf{6 5}$ & $\mathbf{7 0}$ & $\mathbf{7 5}$ & $\mathbf{8 0}$ & $\mathbf{8 5}$ & $\mathbf{9 0}$ & $\mathbf{9 5}$ \\
\cline { 2 - 11 } $\mathbf{5 0}$ & 1.00 & 1.00 & 1.00 & 0.99 & 0.99 & 1.00 & 1.00 & 1.01 & 1.01 & 1.01 \\
$\mathbf{5 5}$ & 1.00 & 1.00 & 0.99 & 0.99 & 0.99 & 0.99 & 1.00 & 1.01 & 1.02 & 1.02 \\
$\mathbf{6 0}$ & 1.00 & $\mathbf{1 . 0 0}$ & 0.99 & 0.99 & 0.99 & 0.99 & 0.99 & 1.01 & 1.02 & 1.03 \\
$\mathbf{6 5}$ & 1.00 & $\mathbf{1 . 0 0}$ & $\mathbf{0 . 9 9}$ & 0.99 & 0.98 & 0.98 & 0.99 & 1.00 & 1.02 & 1.04 \\
$\mathbf{7 0}$ & 1.00 & $\mathbf{1 . 0 0}$ & 0.99 & 0.99 & 0.98 & 0.98 & 0.98 & 0.99 & 1.02 & 1.05 \\
$\mathbf{7 5}$ & 1.00 & 1.00 & 1.00 & 0.99 & 0.99 & 0.98 & 0.97 & 0.98 & 1.01 & 1.05 \\
$\mathbf{8 0}$ & 1.00 & $\mathbf{1 . 0 0}$ & 1.00 & 1.00 & 1.00 & 0.99 & 0.97 & 0.96 & 0.98 & $\mathbf{1 . 0 2}$ \\
$\mathbf{8 5}$ & 1.00 & 1.01 & 1.01 & 1.01 & 1.01 & 1.01 & 0.99 & 0.97 & 0.95 & 0.98 \\
$\mathbf{9 0}$ & 1.00 & 1.01 & 1.01 & 1.02 & 1.03 & 1.03 & 1.02 & 0.99 & 0.95 & 0.93 \\
$\mathbf{9 5}$ & 1.00 & 1.01 & 1.02 & 1.03 & 1.04 & 1.05 & 1.05 & 1.02 & 0.96 & 0.90 \\
\hline
\end{tabular}


Distribution

Function

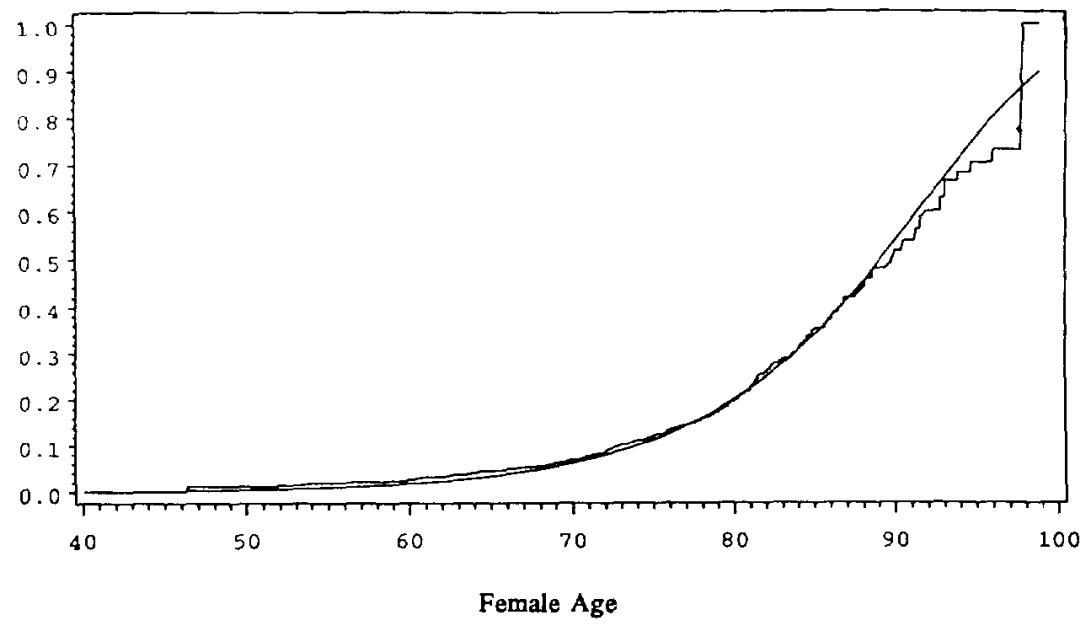

Figure F,1. Gompertz and Kaplan-Meier Fitted Female Distribution Functions. The Gompertz curve is smooth, the Kaplan-Meier is jagged. The distribution is conditional on survival to age forty.

Distribution

Function

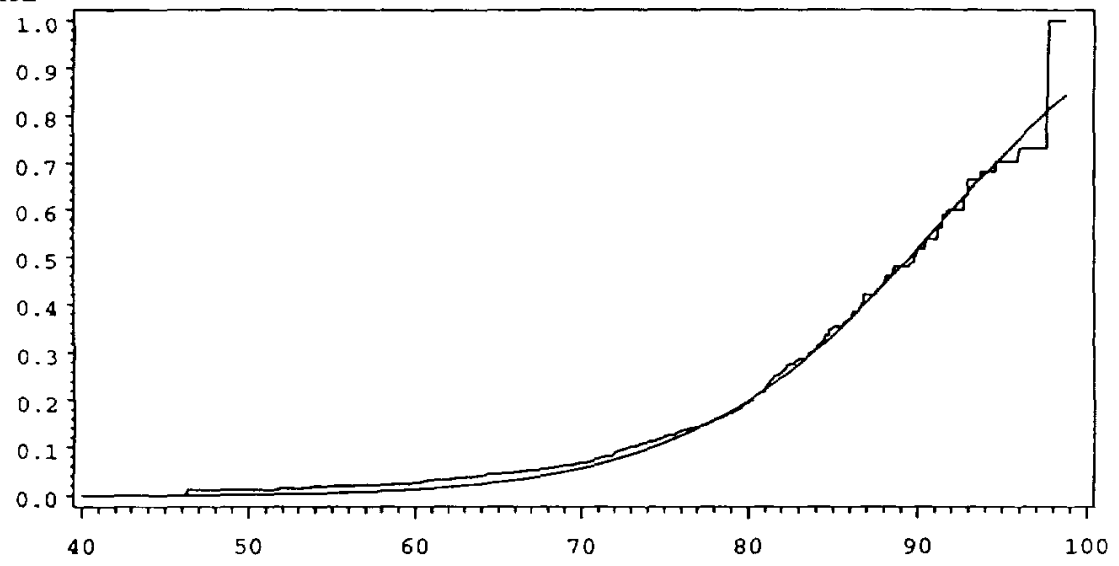

Female Age

Figure F.2. Weibull and Kaplan-Meier Fitted Female Distribution Functions. The Weibull curve is smooth, the Kaplan-Meier is jagged. The distribution is conditional on survival to age forty. 


\section{Distribution}

Function

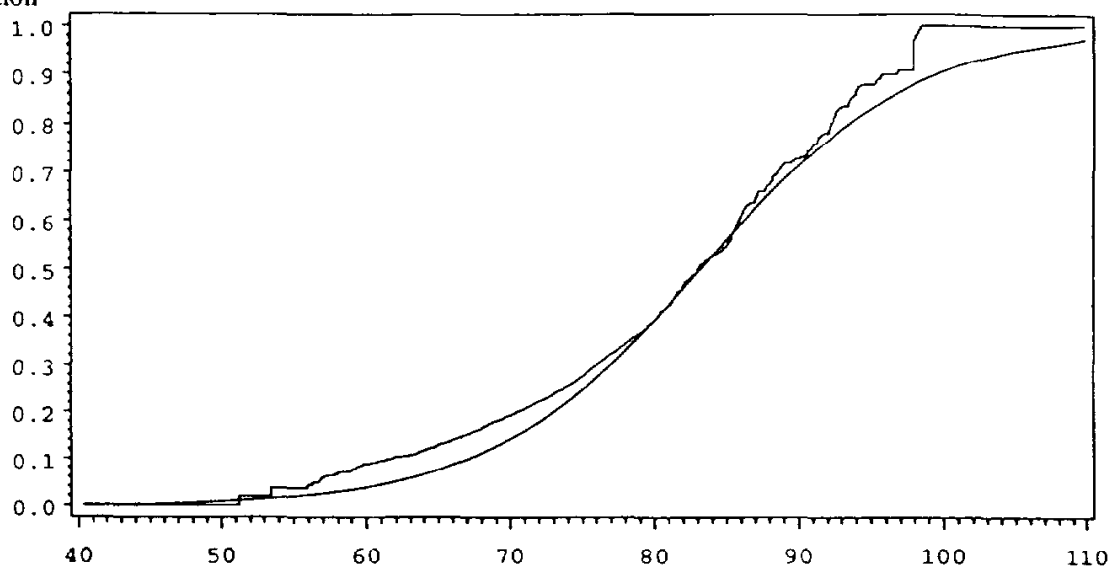

Male Age

Figure F.3. Logistic and Kaplan-Meier Fitted Male Distribution Functions. The Logistic curre is smooth, the Kaplan-Meier is jagged. The distribution is conditional on survival to age forty.

\section{Distribution}

Function

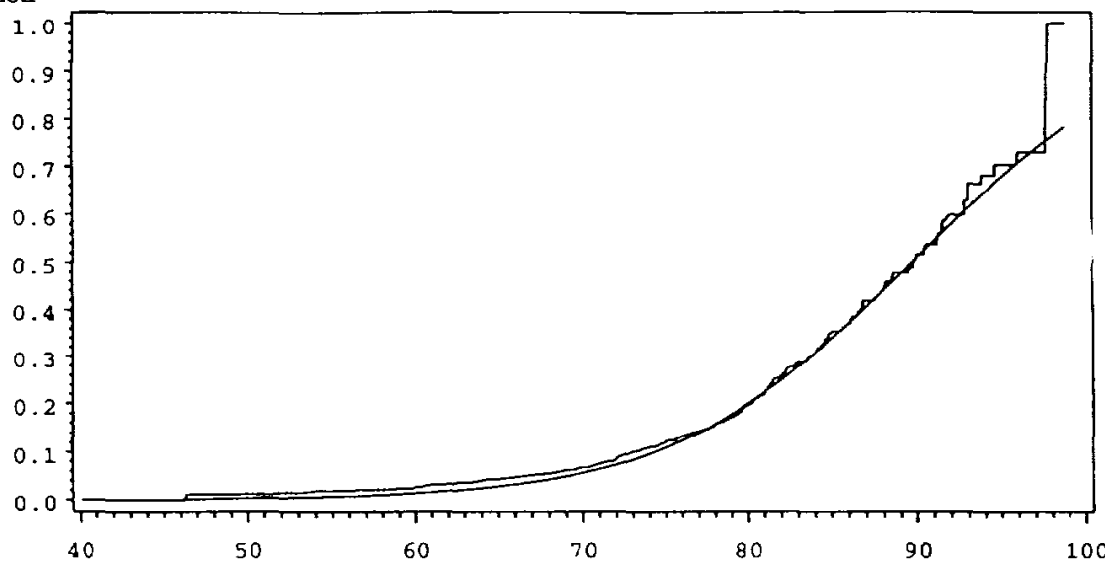

Female Age

Figure F.4. Logistic and Kaplan-Meier Fitted Female Distribution Functions. The Logistic curve is smooth, the Kaplan-Meier is jagged. The distribution is conditional on survival to age forty. 